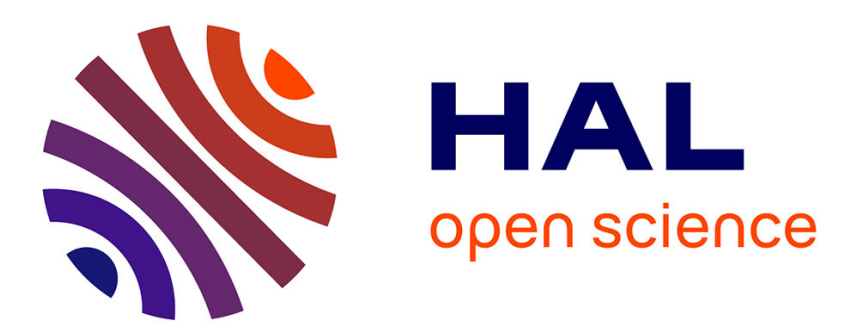

\title{
A perception-oriented Markov model of loss incidents observed over VoIP networks
}

\author{
Sofiene Jelassi, Gerardo Rubino
}

\section{To cite this version:}

Sofiene Jelassi, Gerardo Rubino. A perception-oriented Markov model of loss incidents observed over VoIP networks. Computer Communications, 2018, 128, pp.80-94. 10.1016/j.comcom.2018.06.009 . hal-01962917

\section{HAL Id: hal-01962917 https://hal.inria.fr/hal-01962917}

Submitted on 20 Dec 2018

HAL is a multi-disciplinary open access archive for the deposit and dissemination of scientific research documents, whether they are published or not. The documents may come from teaching and research institutions in France or abroad, or from public or private research centers.
L'archive ouverte pluridisciplinaire HAL, est destinée au dépôt et à la diffusion de documents scientifiques de niveau recherche, publiés ou non, émanant des établissements d'enseignement et de recherche français ou étrangers, des laboratoires publics ou privés. 


\title{
A Perception-Oriented Markov Model of Loss Incidents observed over VoIP Networks
}

\author{
Sofiene Jelassi*, Gerardo Rubino \\ Irisa/Inria, Campus Universitaire de Beaulieu, 35042 Rennes Cedex, France
}

\begin{abstract}
Markov models of loss incidents happening during Packet Voice Communications, a.k.a. Markov loss models, are needful for many engineering tasks, namely network dimensioning and automatic quality assessment. Two very simple ones are Bernoulli and 2-state Markov models, but they carry limited information about incurred loss incidents. On the other hand, a general Markov loss model including $2^{k}$ states, where $k$ is the window length used for observing the voice packet arrival process is characterized by an intractable modeling complexity and an excessive lookahead delay. Moreover, legacy Markov loss models concentrate mostly on capturing some physical characteristics of loss incidents, rather than their perceived effects.

This paper proposes a comprehensive and rather detailed Markov loss model considering the distinguished perceived effects caused by different loss incidents. Specifically, it explicitly differentiates between (1) isolated 20 msec loss incidents which are inaudible by the human ears, (2) highly and lowly frequent short loss incidents $(20-80 \mathrm{msec})$ that are perceived by humans as bubbles and (3) long loss incidents ( $\geq 80 \mathrm{msec}$ ) inducing in-
\end{abstract}

\footnotetext{
${ }^{*}$ Corresponding author

Email addresses: Sofiene.Jelassi@irisa.fr (Sofiene Jelassi), Gerardo.Rubino@inria.fr (Gerardo Rubino)
} 
terruptions that dramatically decrease speech intelligibility. Our numerical analysis show that our Markov loss model captures subtle characteristics of loss incidents observed in empirical traces sampled over representative network paths.

Keywords: packet voice communications, packet loss distribution, Markov loss models, loss-related distortions.

\section{Introduction}

A statistical characterization of loss incidents observed during Packet Voice Communications (PVC) is essential for assessing automatically their perceived quality $<1>$. The study of loss incidents and their resulting distributions has been done while the Internet architecture and traffic were evolving $\langle 2$, 3; , 4; 5, 6, 7, 8, 9; 10, 11 $>$. Today, loss incidents are still a persisting problem, even for well-engineered and provisioned networks $\langle 12>$. Indeed, loss incidents are still occurring not only because of network congestion $\langle 11\rangle$ but also due to routing pathologies $\langle 13\rangle$, traffic anomalies $\langle 14>$, node and link failures $\langle 11>$, and users' mobility $\langle 15$; $16>$.

The measurement studies of worldwide PVC revealed that packet loss processes involve different types of loss incidents. We note that a "loss incident" is defined in our work as a single lost packet or a sequence of consecutively dropped ones, preceded and succeeded by successfully played packets. The perceived distortions are closely related to the number of packets included in the observed loss incidents in addition to their distribution $\langle$ 7; 10$\rangle$. In $\langle 2>$, the authors found that (a) loss incidents are mostly "isolated", i.e., they include a single packet where preceding and succeeding loss incidents are temporally distant from the current one; (b) short loss incidents, including 2-4 consecutive missing packets, are frequently observed; 
(c) long loss incidents, including more than 5 consecutive missing packets, happen from time to time, but are finally regularly observed at each measurement site; (d) the resulting average of loss incident length is very small. It is currently admitted that lost packets are temporally dependent, meaning that the probability of losing a packet is higher if the previous ones were lost, and reciprocally $\langle 2$; $4>$. It is presumed that long loss incidents come mostly from a transient loss of connectivity, as argued in $\langle 8,11$; $9>$. The resulting perceived effects of different types and patterns of loss incidents have been basically studied by scientists working in the auditory perception field $\langle 17>$. The interesting observations related to PVC are the following: (1) periods of isolated loss incidents resulting in a voice gap less than $20 \mathrm{msec}$ are inaudible by users because they are well recovered by PLC (Packet Loss Concealment) algorithms; (2) periods of short loss incidents causing a gap in the order of 40-80 msec are perceived by users as bubbles where intelligibility of rendered voice stream is preserved; (3) periods of long loss incidents resulting in a gap higher than 80 msec induce a clearly perceived interruption in the rendered speech stream and a reduction of its intelligibility and, as a consequence, (4) packet loss ratio (PLR) and/or average length of loss incidents are insufficient in order to accurately reflect the overall degradation level $<1>$. All these observations should be formally specified and normalized. They must be considered for characterizing loss incident distributions in order to faithfully quantify the packet loss-induced distortions, and hence the overall perceived quality.

Typically, distributions of loss incidents are characterized using discrete time Markov models. They are preferred to direct metrics sampling, because of their expressiveness and efficiency. In fact, they are widely-used by the research community to study different aspects of the related communication 
systems. These models rely on well-understood and popular mathematical concepts enabling a straightforward analysis and interpretation. Moreover, they may be used for quality monitoring, network dimensioning and traffic prediction. They should be calibrated in order to be used in monitoring degradations caused by loss incidents. At various quality sampling instants, suitable quality-degradation metrics are retrieved from the calibrated models. They are given as inputs to the perceived quality mapping functions, which transform those metrics into opinion scores. The key challenge is to build a Markov model of loss incidents, a.k.a. a Markov loss model, that is able to capture all metrics required to quantify distortions caused by loss incidents. As we show in next section, besides the general Markov loss model, today there is no comprehensive loss model satisfying that requirement. This is the main concern addressed in our paper.

The main contribution of our work is the proposal of a Markov loss model capturing key metrics that enable the characterization of the perceived degradations caused by different distributions of loss incidents. To do that, we identified and defined four types of loss incident periods resulting in distinguished perceived distortions of PVC. Each loss period was modeled by a Discrete-Time Markov Chain (DTMC). The four resulting Markov models are interconnected using specific entry/exit states. The variation of perceived quality at the communication channel was considered by aggregating defined loss incident periods into GOOD and BAD "macro" states. The proposed loss model was built using the notion of "slot", that is, the atomic time unit used in order to process speech frames. Thus, the operational layout of our loss model changes according to the relationship between packet and slot intervals. The on-line calibration of our loss model was realized thanks to very efficient and deterministic classification algorithms 
of observed loss incident periods. Numerical examples show that our loss model follow accurately behaviors of loss incidents observed over a representative set of network paths. It is worth noting here that our loss model may be used for (1) generating realistic PVC streams observed over current transport networks that enables to understand and evaluate the behavior and performance of voice applications and (2) accurately characterizing and measuring quality of PVC over a today network for better control and management.

The remaining of our paper is organized as follows. Section 2 reviews existing models used to characterize loss incident processes. Section 3 describes our perception-oriented Markov loss model. The classification and calibration algorithms are presented in Section 4. We discuss numerical results and observations in Section 5. We conclude in Section 6 with a discussion about the paper and the related research perspectives.

\section{The state of the art}

There are many discrete time Markov models proposed in the literature for capturing and reproducing the behavior of loss incidents observed within PVC. We are going to survey them using the notation given in Table 1. Two key variables will be used hereafter; LLI and ILD referring respectively to the Length of Loss Incidents and the Inter-Loss Distance expressed in term of voice chunks that may be either packet or slot.

Note first that loss models of order $k$ refer to those that explicitly consider the temporal dependency between a given lost packet and the $k$ preced-

ing ones. The general loss model of order $k$ includes $2^{k}$ states, representing all combinations of $k$ consecutive loss statuses. This model has been ex- 
plored in $\langle 18$; 5$\rangle$, which showed that an order less than 6 was required for cases where the packet interval was set to $160 \mathrm{msec}<\underline{5}>$. However, it was found that an order ranging between 10 and 40 was required for cases where the packet interval was set to $20 \mathrm{msec}$ and $40 \mathrm{msec}$. Its main drawback, for our purposes, comes from the fact that it is physical-oriented rather than perception-oriented. Moreover, a high order value results in intractable modeling complexity, high calibration overhead, and excessive lookahead delay $\langle\underline{4}, \underline{6}>$.

At order 0, the Markov loss model corresponds to the Bernoulli model where packet losses are independent and identically distributed. In such a case, the packet loss distribution is characterized using one single parameter, $p_{u}$, the loss probability, or loss ratio $<2>$. It is represented in Fig. 1(a) using a 2-state Markov chain, where as stated above, $p_{u}=p_{c}$. This metric has been adapted by $\mathrm{R}$. Koodli et al. in order to only consider noticeable losses inducing perceived distortions $\langle\underline{3}>$. As such, a missing packet is considered as concretely lost if the distance separating it from the previous missing one is smaller than a given threshold $\delta$ that ranges between $80 \mathrm{msec}$ and 320 msec. The noticeable packet loss ratio, $p_{n}$, is equal to the ratio between the number of noticeable losses and the number of sent packets. These loss models are definitely unsuitable for capturing the temporal dependency of lost packets observed over VoIP networks $\langle 4,6$, 2, 2, 18, 5>. This is a critical deficiency since that aspect influences considerably the resulting perceived quality of a PVC. At order 1, the general Markov loss model is reduced to a 2-state chain, known as the simple Gilbert loss model $\langle 19>$. It is composed of the NO-LOSS and LOSS states, where all packets are transmitted for NO-LOSS state and dropped for LOSS state (see Fig. 1(b)]. It is specified, for instance, using two independent parameters, $p_{u}$ and $\overline{L L I}$ defined in 


\begin{tabular}{|c|c|c|c|}
\hline & & Label & Significance \\
\hline & \multirow{11}{*}{ כ. } & $m$ & The number of DTMC states \\
\hline & & $k$ & The order of the general Markov loss model \\
\hline & & $q$ & The number of transition probabilities \\
\hline & & $n$ & The number of independent parameters of a given DTMC \\
\hline & & $N$ & The number of packets included in a trace \\
\hline & & $T$ & The packet interval in msec \\
\hline & & $\mu$ & The speech time slot in msec \\
\hline & & $Q$ & The number of loss and inter-loss couples \\
\hline & & $\delta$ & The minimal inter-loss time-interval beyond it a loss is unnoticeable \\
\hline & & $\tau$ & The maximum delay beyond it a loss is seen as an interruption \\
\hline & & $\lambda$ & The lost duration beyond it losses are not differentiable \\
\hline \multirow{17}{*}{$\frac{\underbrace{n}_{n=0}}{\underbrace{0}_{n}}$} & \multirow{6}{*}{ 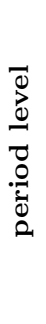 } & $f_{s}, b_{s}$ & The loss frequency of 20 msec-speech slot and the mean loss incident \\
\hline & & & duration during isolated loss periods \\
\hline & & $f_{l}, b_{l}$ & The loss frequency of 20 msec-speech slot and the mean loss incident \\
\hline & & & duration during lowly frequent loss incident periods \\
\hline & & $f_{h}, b_{h}$ & The loss frequency of $20 \mathrm{msec}-\mathrm{speech}$ slot, the mean loss incident \\
\hline & & & duration during highly frequent loss incident periods \\
\hline & \multirow{5}{*}{ 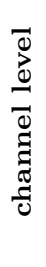 } & & The GOOD/BAD channel status \\
\hline & & $\pi_{g}, \pi_{b}$ & The probability of being in GOOD and BAD channel states \\
\hline & & $p_{g}, p_{b}$ & The packet loss densities in the GOOD and BAD channel states \\
\hline & & $t_{g}, t_{b}$ & The mean periods of GOOD and BAD channel states \\
\hline & & $f_{i}, t_{i}$ & The frequency of interruptions and its mean period \\
\hline & \multirow{6}{*}{$\begin{array}{l}\overline{0} \\
\stackrel{0}{0} \\
= \\
\overline{0} \\
\frac{0}{0} \\
\frac{0}{\infty}\end{array}$} & $p_{u}$ & The unconditional packet loss ratio \\
\hline & & $p_{n}$ & The noticeable packet loss ratio \\
\hline & & $p_{c}$ & The packet loss probability given that the previous one is lost \\
\hline & & $\overline{L L I}, \overline{I L D}$ & The mean values of LLI and ILD variables \\
\hline & & $V_{\mathrm{LLI}}, V_{\mathrm{ILD}}$ & The variances of LLI and ILD variables \\
\hline & & $\mathrm{CDF}(\mathrm{LLI}), \mathrm{CDF}(\mathrm{ILD})$ & The cumulative distribution functions of LLI and ILD variables \\
\hline
\end{tabular}

Table 1: Notations 
Table 1. The transition probabilities in the simple Gilbert Markov model, $a$ and $b$, are given by $a=p_{u} /\left(1-p_{u}\right)$ and $b=1 / \overline{L L I}$. It was indicated in $\langle 2\rangle$ and confirmed in $\langle\underline{6}\rangle$ that the simple Gilbert model captures well short loss incidents, but does not properly account for long ones and the variation of loss intensity. The nominal Gilbert loss model is an extension of previous model. In the given representation, the Markov chain is the same (see Fig. 1(c)] . The difference is that when in the BAD model, for each arrival, a new and independent Bernoulli variable with parameter $p_{b}$ is drawn, and with probability $p_{b}$ the packet is effectively lost, and consequently, with probability $1-p_{b}$ it is delivered. In any case, the change of state is always governed by the Markovian transitions (rates $b$ and $1-b$ ). The Gilbert-Elliot loss model is an extension of the nominal Gilbert loss model. Now, when in state GOOD (resp. BAD), packets are effectively delivered with probability $1-p_{g}$ (resp. $1-p_{b}$ ) and lost with the respective complementary probabilities. The parameter $p_{g}$ is added in order to account for isolated losses observed in the GOOD state. A limitation of the Gilbert and the Gilbert-Elliot models resides in the fact that isolated losses are poorly considered $\langle\underline{19}>$. Moreover, they are absolutely unable to capture the distributions of ILD and LLI variables.

There are some proposals for reducing the $2^{k}$ state space of the general Markov loss model, while capturing perceptually-important metrics characterizing packet loss distributions. A few of them are discussed below:

1. The extended Gilbert loss model: it is a Markov loss model composed of $m+1$ states, where only one state is used to capture successfully transmitted packets $\langle 4\rangle$. The remaining $m$ states are used to capture the number of packets included in the observed loss incidents. More 


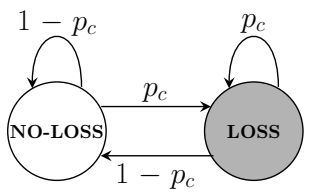

(a) The Bernoulli loss model; $p_{u}=p_{c}$

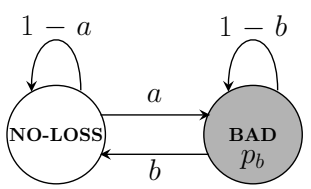

(c) The Gilbert loss model; parameters are: $p_{u}, \overline{L L I}$ and $p_{b}$; the transition probabilities $a$ and $b$ are as in the Gilbert loss model

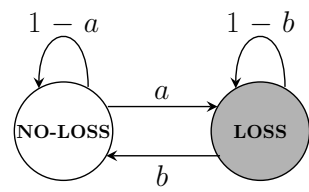

(b) The simple Gilbert loss model; parameters are $p_{u}$ and $\overline{L L I}$; the transition probabilities are $a=p_{u} /\left(1-p_{u}\right)$ and $b=1 / \overline{L L I}$

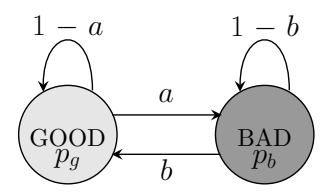

(d) The Gilbert-Elliot loss model; parameters are: $p_{u}$, $\overline{L L I}, p_{g}$ and $p_{b}$; the transition probabilities $a$ and $b$ are as in the Gilbert loss model

Figure 1: The classical Markov loss models at the orders 0 and 1 (notation is given in Table 1) 
precisely, the stationary probability of a state $j$ corresponds to the chance of getting a LLI higher than $j$ packets. This information is crucial for estimating loss-related distortions of PVC. However, the model is unable to capture the features of the ILD distribution, as argued in $\langle 66$, and the variation of loss intensity.

2. The Clark loss model: it is a 4-state Markov model designed to explicitly capture isolated and consecutive packet losses $\langle 4$; 19 $>$. It is an aggregation of 2 simple Gilbert models parameterized in order to reflect GOOD and BAD conditions. The transition probability from LOSS to NO-LOSS is equal to 1 in GOOD conditions. This means that the probability of observing consecutive packet losses in GOOD conditions is zero. Even though it gives better accuracy than the previously described 2-state Markov loss model, the model is unable to carry accurate information about the distributions of LLIs and ILD variables.

3. The Calafate loss model: it is a 3-state Markov loss model that explicitly considers short- and long-time failures of communicating paths, inducing respectively short and long loss incidents $\langle 20>$. To do that, two LOSS states are specified; the first (resp. second) one is used to consider short (resp. long) loss incidents. This information is crucial in order to quantify accurately loss-induced distortions, as discussed earlier. A subjective-based threshold is chosen to classify a loss incident as short or long. This loss model includes a GOOD state where packets are transmitted with probability $1-p_{g}$. The main drawback of this loss model resides in its inability to capture ILD distributions and isolated losses. 


\begin{tabular}{|c|c|c|c|}
\hline Model & $\mathbf{m}$ & $q$ & $\mathbf{n}$ \\
\hline Bernoulli $\langle 2\rangle$ & 1 & 0 & 1 \\
\hline Koodli $\langle 3\rangle$ & 1 & 0 & 1 \\
\hline Gilbert $<2>$ & 2 & 4 & 3 \\
\hline Gilbert-Elliot $\langle 19\rangle$ & 2 & 4 & 4 \\
\hline Calafate $\langle 20\rangle$ & 3 & 7 & 4 \\
\hline Clark $\langle 7>$ & 4 & 8 & 5 \\
\hline Sanneck $\langle 4\rangle$ & $\mathrm{m}$ & $2 \mathrm{~m}+1$ & $\mathrm{~m}+1$ \\
\hline General $<18>$ & $2^{k}$ & $2^{2 k-1}$ & $2^{2 k-1}$ \\
\hline
\end{tabular}

Table 2: The characteristics of Markov loss model capturing packet loss distribution

\begin{tabular}{|c|c|c|c|c|c|c|c|c|c|c|c|c|}
\hline Model & $p_{u}$ & $p_{n}$ & $\overline{I L D}$ & $\overline{L L I}$ & $p_{c}$ & $V_{\text {LLI }}$ & $f_{l}$ & $f_{h}$ & $f_{i}$ & $s$ & $\pi_{g}$ & $\pi_{b}$ \\
\hline Bernoulli $\langle 2>$ & $\checkmark$ & $x$ & $\times$ & $x$ & $\times$ & $x$ & $x$ & $\times$ & $x$ & $x$ & $\times$ & $\times$ \\
\hline Koodli $\langle 3\rangle$ & $\checkmark$ & $\checkmark$ & $x$ & $\times$ & $x$ & $x$ & $\times$ & $\times$ & $x$ & $x$ & $\times$ & $x$ \\
\hline Gilbert $\langle 2\rangle$ & $\checkmark$ & $x$ & $\checkmark$ & $\checkmark$ & $\checkmark$ & $\times$ & $\times$ & $\times$ & $x$ & $x$ & $\times$ & $\times$ \\
\hline Gilbert-Elliot $\langle 19\rangle$ & $\checkmark$ & $\times$ & $\checkmark$ & $\checkmark$ & $\checkmark$ & $\times$ & $\times$ & $\times$ & $x$ & $\checkmark$ & $\checkmark$ & $\checkmark$ \\
\hline Calafate $\langle 20\rangle$ & $\checkmark$ & $\times$ & $\checkmark$ & $\checkmark$ & $\checkmark$ & $\times$ & $\times$ & $\times$ & $\checkmark$ & $x$ & $\times$ & $\times$ \\
\hline Clark $\langle 7>$ & $\checkmark$ & $\checkmark$ & $\checkmark$ & $\checkmark$ & $\checkmark$ & $\times$ & $\checkmark$ & $\times$ & $x$ & $\checkmark$ & $\checkmark$ & $\checkmark$ \\
\hline Sanneck $\langle 4>$ & $\checkmark$ & $\times$ & $\checkmark$ & $\checkmark$ & $\checkmark$ & $\checkmark$ & $x$ & $\times$ & $\checkmark$ & $x$ & $\times$ & $x$ \\
\hline General $\langle 18>$ & $\checkmark$ & $\checkmark$ & $\checkmark$ & $\checkmark$ & $\checkmark$ & $\checkmark$ & $\checkmark$ & $\checkmark$ & $\checkmark$ & $\checkmark$ & $\checkmark$ & $\checkmark$ \\
\hline
\end{tabular}

Table 3: The capacity of existing models to capture some characterizing metrics of loss distribution on VoIP network 
The characteristics of existing loss models are summarized in Table 2 . The loss-characterizing metrics captured by each loss model are given in Table 3. As we can see, only the generic Markov loss model is able to capture all quality-influencing metrics. However, its high number of states and transition probabilities results in complex and intractable formulas to express them. Moreover, the generic loss model requires a large training dataset to obtain transition probabilities that are roughly independent of time. On the other hand, Markovian loss models with reduced state spaces often enable elaborating simple expressions of different loss-related metrics. Moreover, calibration may be realized in a very efficient way. However, they are able to only capture a limited set of metrics. Thus, our primary goal is to design a reduced-state Markov loss model enabling to capture all previously discussed aspects of loss incidents.

\section{Markov loss model design and its analysis}

In this section, we enumerate the requirements that our loss model should fulfill. Then, we define different loss periods and we describe our loss model that is analyzed in the succeeding section.

\subsection{Markov loss model requirements}

This section enumerates the specifications that should be respected by our sought perception-centric Markov loss model.

1. The model design should account for the packet interval $(T)$ that defines the speech duration carried in a packet. This helps in estimating the number of phonemes - the smallest speech unit comprehensible by 
users - included in a packet. This information is important for evaluating the perceived effects caused by missing packets. Basically, the duration of a phoneme belongs to the interval of 20-100 msec; it depends on the speaker rate and the phoneme characteristics $\langle 10$, 17; 4 $>$. Hence, a missing packet with $T$ value smaller than 20 msec results in removing only a fraction of a phoneme that may be easily restored by human ears $\langle 17\rangle$. However, a missing packet with $T$ value between 20 msec and 100 msec may lead to the loss of a whole phoneme, thus reducing to a certain extent the speech intelligibility $\langle 17\rangle$. A missing packet with $T$ value higher than 100 msec results in removing consecutive phonemes causing severe degradations to speech intelligibility.

2. The model should explicitly differentiate between isolated, short and long loss incidents Moreover, it should discriminate between different frequencies of short loss incidents. Indeed, induced perceived distortions may considerably vary as a function of loss incident frequency, which should be adequately quantified.

3. The model should account for short- and long-term loss behaviors shaping respectively the distribution of lost packets and the overall perceived quality.

\subsection{The loss model design}

Existing Markov loss models are built using a specific packet interval, meaning that elaborated metrics become obsolete once that value changes $\langle 21\rangle$. Note that the packet interval depends of each implementation and may change during a voice session $<22$; $23>$. In order to build packet intervalindependent Markov loss models, we decided to use the concept of slot as a unified measuring unit of voice stream timeline. 
A slot includes data required to play voice for a given time interval, denoted $\mu$. Hence, each voice packet should be transformed into the corresponding number of slots depending on their durations. The selection of the slot duration should account for the speech structure and features in addition to commonly observed packet intervals. Those requirements are fulfilled by choosing a slot interval less than $20 \mathrm{msec}$, on the one hand, and equal to the "lowest common denominator" of commonly observed packet intervals, on the other hand. That is why we propose setting the value of the slot interval to $10 \mathrm{msec}$.

In order to explicitly distinguish between the different perceived lossincident induced distortions described previously, we define the following four types of loss periods: isolated loss period, highly and lowly frequent short loss incident periods, and finally long loss incident period. The concept of isolated loss periods is given in Definition 1 below, where the constant $\delta$ is used in order to differentiate between noticeable and unnoticeable loss instances. Its recommended value varies from $80 \mathrm{~ms}$ to $320 \mathrm{~ms}$.

Definition 1. An isolated loss period is defined as the longest series of slots where all observed LLI are less than 20 msec and preceding and succeeding ILD of each loss incident are greater than $\delta$.

It is worth noting that Definition 1 implies that the value of the minimal loss frequency, $f_{s}$, is equal to $1 / \delta$ and the maximum $p_{u}$ value is equal to $20 /(\delta+20)$. A robust metric measuring the local loss incident frequency should be defined in order to differentiate between lowly and highly frequent short loss incident periods. In our work, we use the metric given in Eq. (1). It considers loss frequencies observed before and after a given loss incident. 


$$
F_{\text {local }}^{j}=\sum_{i=j-w}^{j+w} \frac{1 \text { (slot } i \text { is lost })}{|j-i|}
$$

where $w$ is the window size, $j$ is the slot identifier or index, and $1(P)$ is the indicator function of the predicate $P$. The highly and lowly frequent short loss incident periods are specified in Definitions 2 and 3 . The values of LLI during lowly frequent short loss incident periods should be less than 20 msec. This constraint assures uniform and homogeneous perceived distortions.

Definition 2. A lowly frequent short loss incident period is defined as the longest series of slots where encountered LLI values are shorter than 20 msec. Moreover, the local loss frequency, $F_{\text {local, }}$, should be less than 1 .

Definition 3. A highly frequent short loss incident period is defined as the longest series of slots where encountered LLI values vary between 20 and 80 msec for any $F_{\text {local }}$ value.

Definition 4. An interruption period corresponds to LLI greater than 100 msec.

Each identified loss period type is modeled using a separate discrete time Markov chain. The following graphical convention are going to be used in order to illustrate our Markov chains: (1) transparent and opaque Markov states refer respectively to a received and lost slot; (2) double and dotted lined Markov states correspond respectively to an entry and exit from/to another type of loss period; (3) the entry and exit states are represented using double dotted lines. At each type of loss period, states are numbered 
from 0 to $k$ that is the total number of states. The transition probabilities are denoted $p_{i, j}^{a}$ where $i$ and $j$ refer to the origin and final states and $a$ is the identifier of the loss period type. A label is assigned to each state in order to show the cumulative ILD or LLI probability it accounts for within a given loss period. Precisely, the stationary probability of a transparent (resp. opaque) state whose label is greater than $i \mu$ is equal to $\operatorname{Pr}(I L D \geq i \mu)$ (resp. $\operatorname{Pr}(L L I \geq i \mu))$.

The Markov chain, $M_{0}$ shown in Fig. 2(a) models loss incidents observed during isolated loss periods. It accounts also for successfully received slots for a time interval greater than $\delta=x \mu$ separating short loss incident and interruption periods, where $x$ is a integer ranging between 16 and 32 . The entry and exit events to/from $M_{0}$ can only be observed at state 0 (see Fig. 2(a)p. The Markov chains $M_{1}$ and $M_{2}$ shown in Fig. 2(b) and Fig. 2(c) consider respectively lowly and highly frequent short loss incident periods as specified in Definitions 2 and 3 .

To precisely characterize perceived distortions caused by interruptions (see Definition 4), we propose using chain $M_{3}$ shown in Fig. 2(d), This model captures the cumulative distribution of observed interruption incidents. The time constant $\tau$ is the granularity used in order to discriminate perceived effects caused by different interruption incident durations. We assume that interruptions greater than $\lambda$ result in equivalent perceived distortions. Note that all transition probabilities between different $M_{i}$ are possible.

The proposed discrete time Markov chain considers short-term and longterm loss behaviors shaping respectively the missing slot distributions and the overall perceived quality. To better exhibit that feature, we rearrange our model as shown in Fig. 3. Basically, we aggregate isolated and lowly frequent short loss incidents chains in one "macro" state named A that 


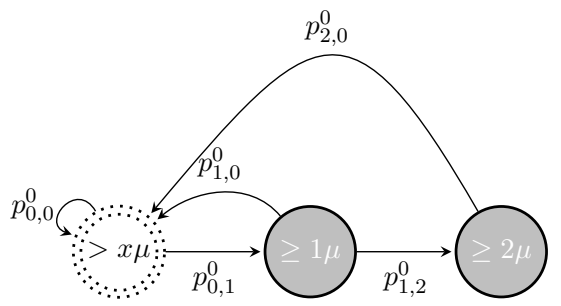

(a) $M_{0}$

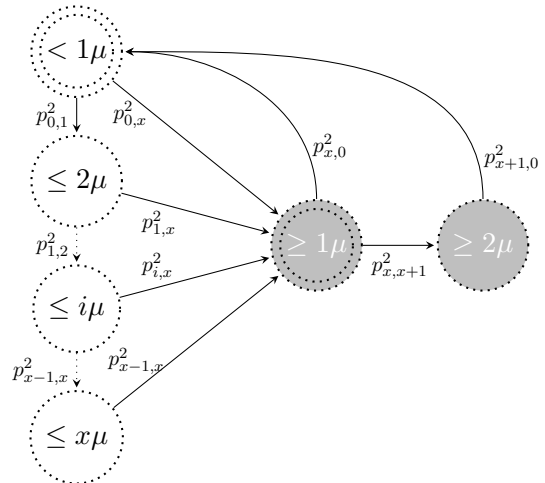

(b) $M_{1}$

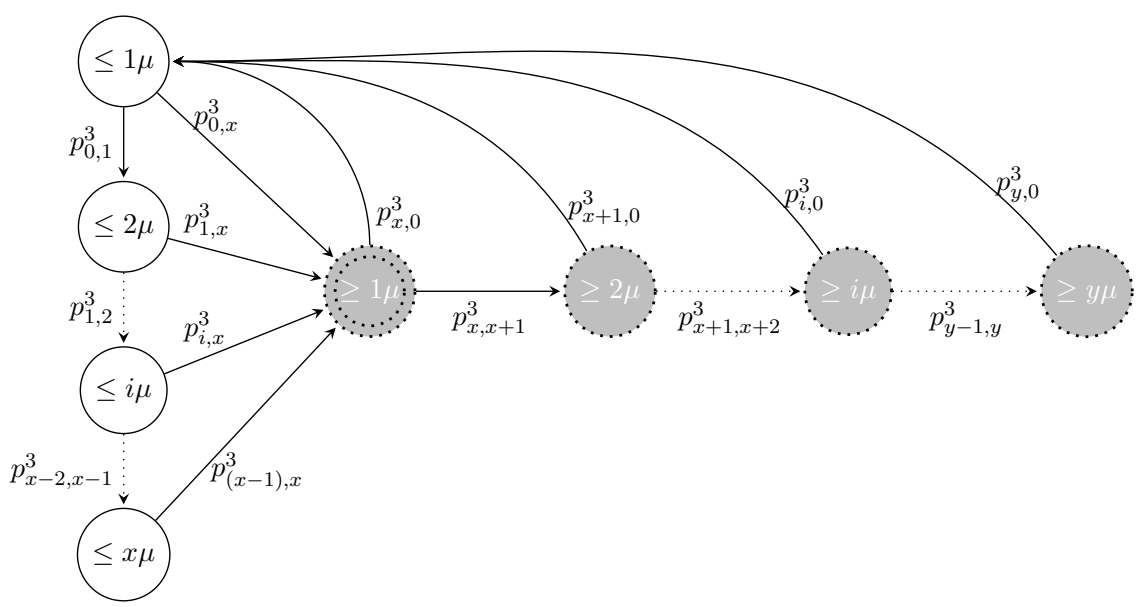

(c) $M_{2}$

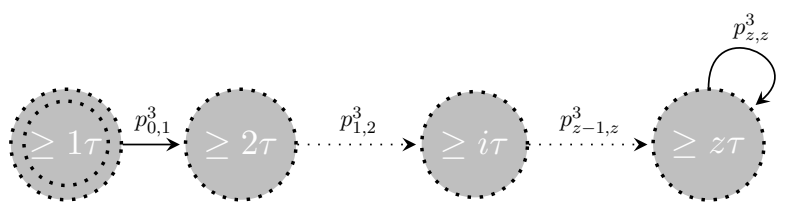

(d) $M_{3}$

Figure 2: The general layout of our Markov loss model 


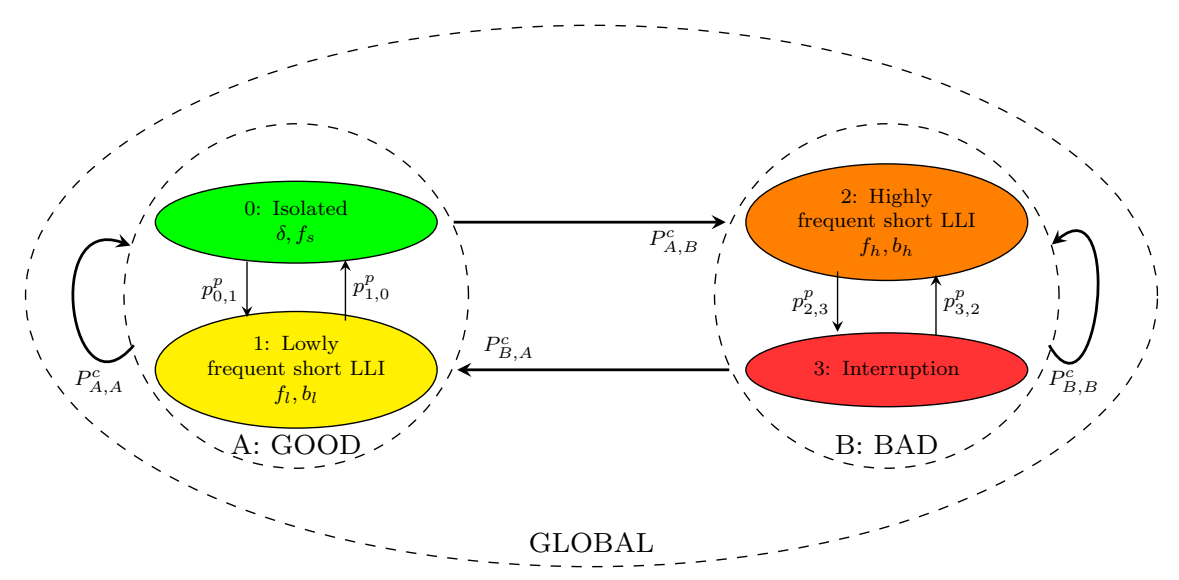

Figure 3: The perception-oriented loss model at channel and region scales

captures channel conditions resulting in GOOD perceived quality. Similarly, we aggregate highly frequent short loss incidents and interruption chains in another "macro" state named B capturing channel conditions resulting in a BAD perceived quality. The transition from macro state $\mathrm{A}$ to macro state $\mathrm{B}$ reflects a switching from GOOD to BAD perceived qualities, and reciprocally (see Fig. 3); it regroups all transitions originating in states of A and terminating in states of $\mathrm{B}$, and inversely. The transition probabilities between macro states (thick arrows in Fig. 3), $P_{A, B}^{c}$ and $P_{B, A}^{c}$, are given in Eq. 2.

$$
P_{A, B}^{c}=\sum_{i \in A} \sum_{j \in B} P_{A} p_{i, j}^{p}, \quad P_{B, A}^{c}=\sum_{i \in B} \sum_{j \in A} P_{B} p_{i, j}^{p}
$$

Here, $P_{A}$ and $P_{A}$ represent respectively the probabilities to be at the macro states $\mathrm{A}$ and $\mathrm{B}$, and $p_{i, j}^{p}$ refers to the transition probability in the loss period chain, from $i$ to $j$.

By doing that final milestone, we end up with a Markov loss model that fulfills all requirements specified in Section 3.1. In next subsection, we are 


\begin{tabular}{c||c|c|c|c||c} 
& $M_{0}$ & $M_{1}$ & $M_{2}$ & $M_{3}$ & $\mathrm{~m}$ \\
\hline $\mathrm{T} \leq 20 \mathrm{msec}$ & 3 & 18 & 24 & 4 & 49 \\
$20 \mathrm{msec}<\mathrm{T} \leq 80 \mathrm{msec}$ & 1 & 0 & 24 & 4 & 29 \\
$80 \mathrm{msec}<\mathrm{T} \leq 160 \mathrm{msec}$ & 1 & 16 & 0 & 4 & 21 \\
$160 \mathrm{msec}<\mathrm{T} \leq 320 \mathrm{msec}$ & 1 & 0 & 0 & 4 & 5 \\
$\mathrm{~T}>320 \mathrm{msec}$ & 1 & 0 & 0 & 1 & 2 \\
\hline
\end{tabular}

Table 4: The actual number of states under each packet interval setting.

going to analyze it in order to explore its features and usage.

\subsection{The Markov loss model analysis}

This section is split into two parts; the first one discusses the relationship between general and active layouts of our Markov loss model; the second one gives expressions enabling to compute various loss metrics given in Table 1 .

The general layout of our Markov loss model is wholly defined as a function of the previously specified time constant tuple $(\mu, \delta, \tau, \lambda)$. The general layout is definitely insensitive to the packet interval, $T$, in opposition to the actual layout, which includes only concretely visited states. As a general rule, the greater the value of $T$, the lower the number of visited states. Table 4 summarizes the number of visited states for different values of $T$ when the time constant tuple $(\mu, \delta, \tau, \lambda)$ is set to $(10 \mathrm{msec}, 160 \mathrm{msec}, 80 \mathrm{msec}$, $320 \mathrm{msec}$ ). As we can see, a value of $T$ greater than $\lambda$ transforms our Markov loss model into a simple Gilbert model. However, a value of $T$ that ranges between $\tau$ and $\lambda$ transforms our Markov loss model into an extended Gilbert model. Finally, a value of $T$ greater than $2 \mu$ and less than $\delta$ leads to chains that are close to Clark models $<7>$.

We will now describe how to use this Markov model in order to compute the loss characterization metrics given in Table 1. Note that Appendix A contains algorithms enabling to compute different loss metrics, directly sam- 


\begin{tabular}{|c|c|c|}
\hline & Metrics & Expression \\
\hline & $\begin{array}{c}p_{0}, p_{1}, p_{2}, p_{3} \\
t_{0}, t_{1}, t_{2}, t_{3} \\
f_{s}, b_{s} \\
f_{l}, b_{l} \\
f_{h}, b_{h}\end{array}$ & $\begin{array}{l}\operatorname{det}\left(R_{k}\right) / \operatorname{det}(R) \\
1 / p_{0}, 1 / p_{1}, 1 / p_{2}, 1 / p_{3} \\
1 /\left[p_{0,0}^{0}+p_{1,0}^{0}+p_{1,2}^{0}\right], \frac{1}{2}\left[\left(p_{1}^{0}-p_{2}^{0}\right) t_{0}^{1}+t_{0}^{2}\right] \\
\frac{1}{x}\left[\sum_{k=2}^{x}\left(p_{k}^{1}-p_{(k-1)}^{1}\right) t_{1}^{k-1}+t_{1}^{x}\right], \frac{1}{2}\left[\left(p_{x}^{1}-p_{x+1}^{1}\right) t_{1}^{x}+t_{1}^{x+1}\right] \\
\frac{1}{x}\left[\sum_{k=2}^{x}\left(p_{k}^{2}-p_{(k-1)}^{2}\right) t_{2}^{k-1}+t_{2}^{x}\right], \frac{1}{y}\left[\sum_{k=2}^{y}\left(p_{k}^{1}-p_{(k-1)}^{1}\right) t_{2}^{k-1}+t_{2}^{y}\right]\end{array}$ \\
\hline & $\begin{array}{l}\pi_{g}, \pi_{b} \\
t_{g}, t_{b} \\
p_{g}, p_{b} \\
f_{i}, t_{i}\end{array}$ & $\begin{array}{l}p_{0}+p_{1}, p_{2}+p_{3} \\
t_{0}+t_{1}, t_{2}+t_{3} \\
\frac{1}{t_{g}}\left(t_{0} p_{0}+t_{1} p_{1}\right), \frac{1}{t_{b}}\left(t_{2} p_{2}+t_{3} p_{3}\right) \\
f_{i}=1 /\left(t_{0}+t_{1}+t_{2}\right), t_{i}=t_{3}\end{array}$ \\
\hline & $\begin{array}{l}V_{\mathrm{ILD}}, V_{\mathrm{LLI}} \\
\mathrm{CDF}(L L I) \\
\mathrm{CDF}(I L D)\end{array}$ & 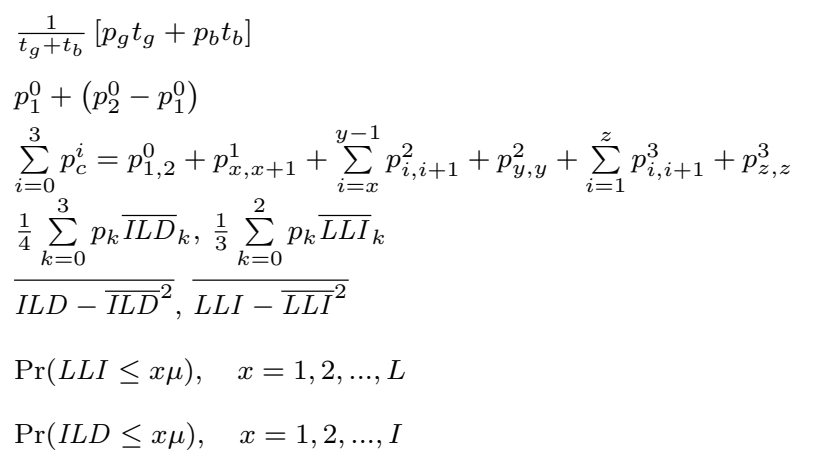 \\
\hline & \multicolumn{2}{|c|}{$\begin{array}{l}\text { Notations and Remarks } \\
\text { det is a function that computes determinant of a matrix } \\
\mathrm{x} \text { and y are respectively equal to }\lfloor\delta / \mu\rfloor \text { and }\lfloor\tau / \mu\rfloor \\
R \text { is the transition matrix of loss periods }(4 \times 4) \\
p_{k}^{i} \text { refers to the stationary probability of state } k \text { included in } M_{i} \\
t_{k}^{i} \text { refers to the mean spent time at state } k \text { included in } M_{i} \\
\overline{I L D}_{k} \text { and } \overline{L L I}_{k} \text { are average lengths of ILD and LLI variables in each loss period }\end{array}$} \\
\hline
\end{tabular}

Table 5: The loss characterization metrics 
pled from a given loss pattern. Here, we assume that all transition probabilities are provided by a counting algorithm that is going to be presented in Section 4. We start the analysis of our proposed Markov loss model by computing the stationary probabilities of each loss period kind $p_{0}, p_{1}, p_{2}$ and $p_{3}$, using the balance equations below.

$$
\begin{aligned}
& p_{0,1}^{p} p_{1}+p_{0,2}^{p} p_{2}+p_{0,3}^{p} p_{3}=p_{0}, \\
& p_{1,0}^{p} p_{0}+p_{1,2}^{p} p_{2}+p_{1,3}^{p} p_{3}=p_{1}, \\
& p_{2,0}^{p} p_{0}+p_{2,1}^{p} p_{1}+p_{2,3}^{p} p_{3}=p_{2}, \\
& p_{3,0}^{p} p_{0}+p_{3,1}^{p} p_{1}+p_{3,2}^{p} p_{2}=p_{3} .
\end{aligned}
$$

The transition probabilities $p_{i, j}^{p}$ between different loss periods' chains are computed as follows:

$$
p_{i, j}^{p}=\sum_{s \in i_{\text {out }}} \sum_{t \in j_{\text {in }}} p_{s}^{i} p_{s, t}^{i, j}
$$

where $i_{\text {out }}$ and $j_{\text {in }}$ sets contain respectively exit and entry states of various loss period' types $i$ and $j$. The mean residence time $t_{i}$ is equal to $1 / p_{i}$ for $i \in$ [1..3]. The expressions for computing mean LLI and ILD metrics under each period type are given in Table 5. In a similar way, the stationary probability of each individual state included in each loss period type can be obtained. The stationary probabilities at GOOD and BAD quality conditions are given by $\pi_{g}=p_{0}+p_{1}$ and $\pi_{b}=p_{2}+p_{3}$. The mean residence times at GOOD and BAD quality conditions are equal to $t_{g}=t_{0}+t_{1}$ and $t_{b}=t_{2}+t_{3}$. The density loss probabilities at GOOD and BAD states are given by: 


$$
\begin{aligned}
& p_{g}=\frac{1}{t_{g}}\left(t_{0} p_{0}+t_{1} p_{1}\right), \\
& p_{b}=\frac{1}{t_{b}}\left(\begin{array}{lll}
t_{2} & p_{2}+t_{3} & p_{3}
\end{array}\right) .
\end{aligned}
$$

The mean interruption duration is equal to $t_{3}$. The mean frequency of observed interruptions is given by: $f_{i}=1 /\left(t_{0}+t_{1}+t_{2}\right)$. The global metrics, $p_{u}$, can be computed as follows:

$$
p_{u}=\sum_{i=0}^{3} p_{i} p_{u}^{i}=p_{0}\left[\frac{t_{0}^{1}+t_{0}^{2}}{t_{0}}\right]+p_{1}\left[\frac{t_{1}^{x+1}+t_{1}^{x}}{t_{1}}\right]+p_{2}\left[\frac{1}{t_{2}} \sum_{i=x}^{y-1} t_{2}^{i}\right]
$$

where $p_{u}^{i}$ is the ratio of missing slots observed at loss period type $i$ and $t_{i}^{j}$ is the mean residence duration at state $j$ belonging to loss period type $i$. The values of $x$ and $y$ are respectively equal to $\lfloor\delta / \mu\rfloor$ and $\lfloor\tau / \mu\rfloor$ (see Fig. 2). The noticeable loss ratio is equal to the loss ratio observed at loss period type 0 , which is given by:

$$
p_{n}=\frac{1}{t_{0}}\left(t_{0}^{1}+t_{0}^{2}\right)
$$

The general expression enabling to compute $p_{c}$, the conditional loss probability, is given in Eq. 9 .

$$
p_{c}=\sum_{i=0}^{3} p_{c}^{i}=p_{1,2}^{0}+p_{x, x+1}^{1}+\sum_{i=x}^{y-1} p_{i, i+1}^{2}+p_{y, y}^{2}+\sum_{i=1}^{z} p_{i, i+1}^{3}+p_{z, z}^{3}
$$

where $z$ is equal to $\lfloor\lambda / \mu\rfloor$. The mean ILD and LLI durations, $\overline{I L D}$ and $\overline{L L I}$, are simply equal to the weighted average of $\overline{I L D}_{i}$ and $\overline{L L I}_{i}$ values observed 
at each loss period, where the weighting coefficients are equal to the corresponding stationary probabilities. The empirical cumulative distributions of LLI and ILD functions are respectively given by:

$$
\begin{aligned}
& -\operatorname{CDF}(I L D)=\operatorname{Pr}(I L D \leq x \mu), \quad x=1,2, \ldots, I \\
& -\operatorname{CDF}(L L I)=\operatorname{Pr}(L L I \leq x \mu), \quad x=1,2, \ldots, L
\end{aligned}
$$

where $I \mu$ and $L \mu$ represent the longest observed ILD and LLI values. The obtained distribution enables easily computing the variance of ILD and LLI variables. Table 5 summarizes all computed metrics using our discrete time Markov model.

\section{The Markov loss chain implementation}

This section presents important aspects related to the implementation of our Markov loss model. First, we describe how to classify loss periods enclosed in a given lost slot distribution. Second, it presents a calibration algorithm to compute and update at runtime the transition probabilities of the loss model.

\subsection{The loss period classifier}

The proposed Markov loss model requires tagging different loss periods enclosed within a given loss distribution. An example of a such operation is given in Fig. 4. The problem of loss period recognition may be formulated as follows: do I need to change or to keep the current loss period once a new $\{$ ILD, LLI $\}$ sample is measured? An event-driven rather than a windowbased technique is better to solve the issue. An excessive waiting time to update our model caused by a long $\{$ ILD, LLI $\}$ sample can be overcome by 


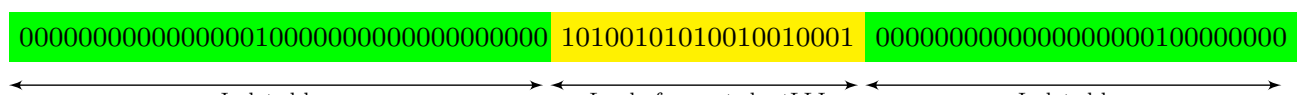

Isolated loss $\longleftrightarrow$ Lowly frequent shortLLI $\longleftrightarrow$ Isolated loss

\begin{tabular}{|c|c|c|}
\hline 1100101100111 & 000001010010100000000000 & 11111100000000000000100001000000001100010100001 \\
\hline
\end{tabular}

Figure 4: A loss period classification of received/lost slot distributions.

trigging update procedure after a given delay, e.g. $0.5 \mathrm{sec}$. The measured ILD and LLI variables contain information regarding the start and end times of the observed events. A rule-based policy is used in order to manage transitions between different detected loss periods.

$\overline{\text { Algorithm } 1 \text { A global algorithm used to tag received/lost slot distributions }}$ Input: A new $\{L L I, I L D\}$ sample or update timeout triggered Output: Recognized type and limits of encountered loss periods

1: if LLI $>\tau$ then

2: Algorithm 2

3: else if lossType = ISOLATED then

4: Algorithm 3

5: else

6: Algorithm 4

7: end if

The main building blocks of our loss period classifier are given in Algorithm 1. As we can see, an interruption incident is recognized if the sampled LLI value is higher than $\tau$ whatever the type of the current loss period. The transition from the prevailing loss period to an interruption is done as a function of its type and the value of ILD variable, as outlined in Algorithm 2. The loss frequency function, LLI-freq-tag(), is used in order to classify short loss incident periods as low or high, following the Definitions 2 and 3 . This function is called at the end of each detected short loss incident periods. 


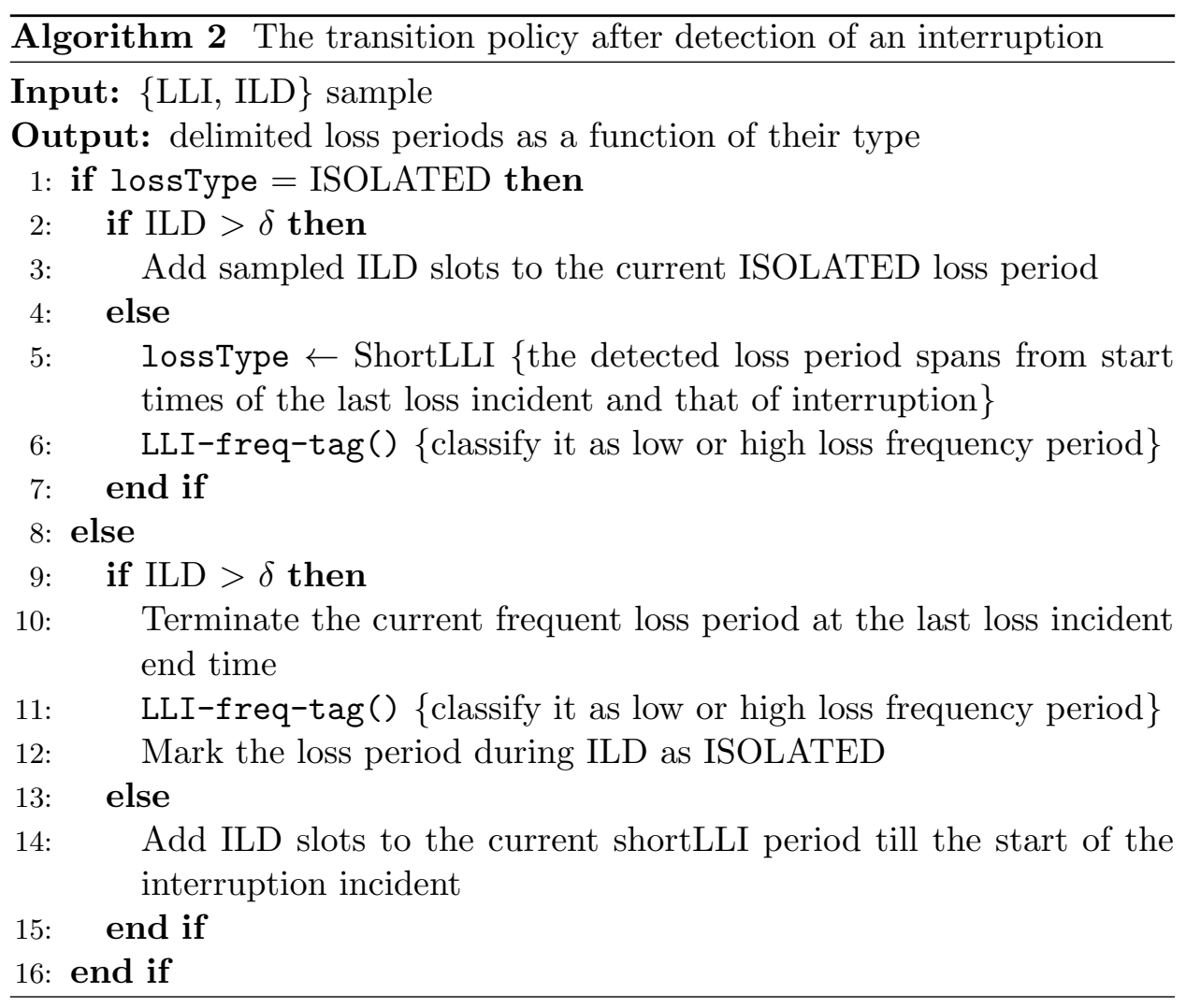


Algorithms 3 and 4 are used when the value of LLI is less than $\tau$ for isolated and short loss incident ongoing periods. As we can see, Algorithm 3 expands a current isolated loss period to enclose previous LLI and ILD slots if and only if the measured ILD value is greater than $\delta$. In such a case, a measured LLI value greater than 20 msec causes a switching to a short loss incident period, initiated from the start time of the measured loss incident. On the other hand, if the value of ILD is less than $\delta$, then a short loss incident period is switched on from the start time of the previous loss incident. Algorithm 4 immediately switches to an isolated loss period when the value of ILD is greater than $\delta$. Otherwise, it expands the short loss incident period to include incoming slots.

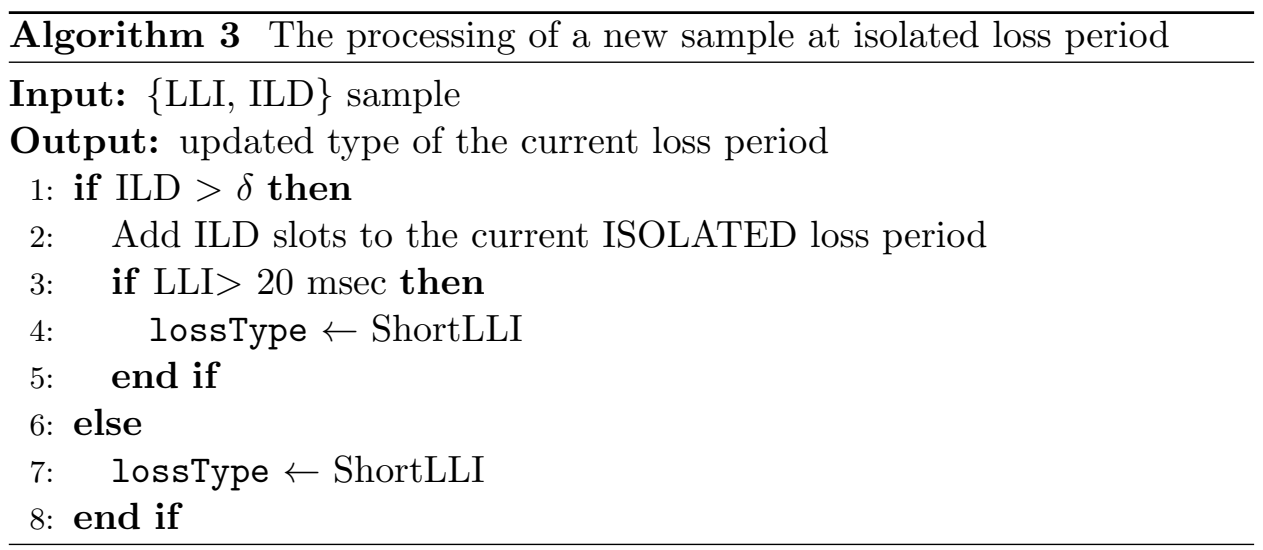

\subsection{The chain calibration algorithm}

The proposed Markov loss model must be calibrated at runtime to characterize observed loss incidents. This is done by updating at regular intervals the transition-probability matrix, $X$, using information added by the loss period classifier. Eq. 10 gives a generic way to compute $X$ elements, referred 


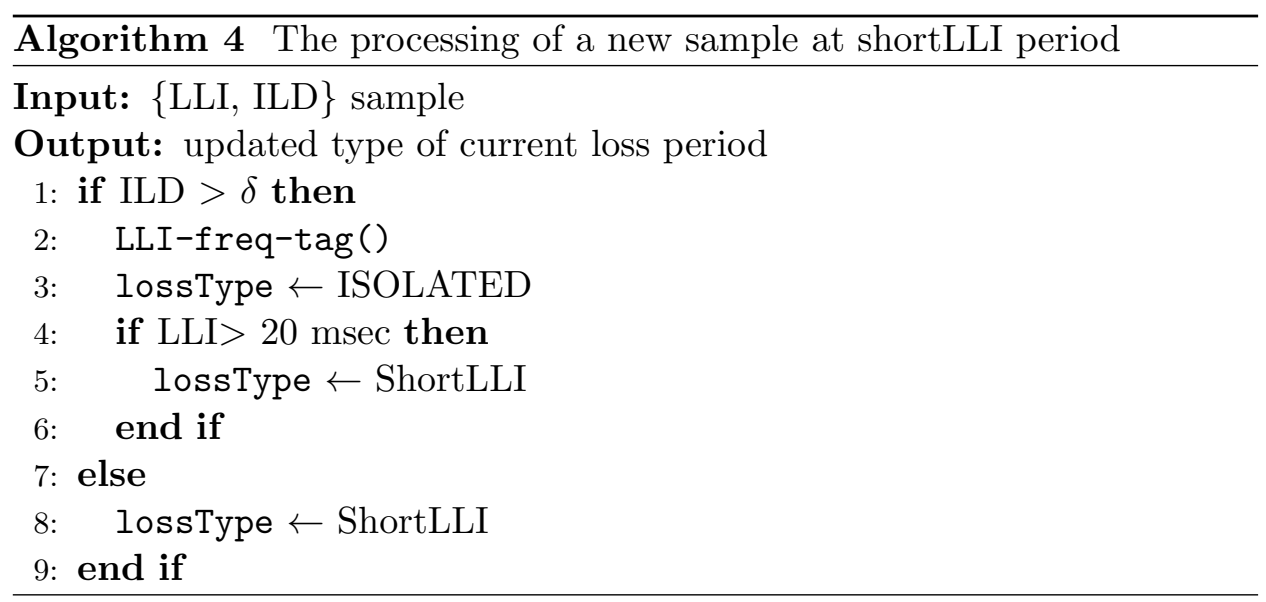

to as $x_{i, j}, 1 \leq i, j \leq m$.

$$
x_{i, j}= \begin{cases}\frac{c_{i, j}}{\sum_{k=1}^{N} c_{i, k}} & \text { if } c_{i, j} \geq 0, \\ 0 & \text { otherwise }\end{cases}
$$

where $c_{i, j}$ is the number of observed transitions from $i$ to $j$ during a monitoring interval. The matrix of counters is updated using a set of rules. They are defined as follows: $\left\{x_{a}, s_{a}, e\right\} \Rightarrow\left\{x_{b}, s_{b}, c_{a, b}\right\}$ where $x_{a}$ and $s_{a}$ refer respectively to the current loss period type and state, $e$ is the read event, $x_{b}$ and $s_{b}$ represent respectively the next loss period and state, and $c_{a, b}$ is a scalar counting the number of times a rule has been visited. More precisely, our counting algorithm executes two steps: (1) it identifies the suitable rule from the examined distribution of received/lost slots and (2) it increments by 1 the corresponding counter. At the end of a monitoring interval the matrix of counters is used in order to compute $X$.

One can maintain instantaneous and overall versions of $X$. The former 
one accounts only for measures realized between two measurements instants that is used for getting short-term loss metrics. The latter is a combination of instantaneous $X$ matrices that is basically used to derive long-term loss metrics.

\section{The numerical analysis of our loss model}

The goals of this section are twofold: (1) identify the minimum length of a collected trace necessary to extract reliable loss metrics from the proposed Markov loss model, and (2) validate the ability of our model to deal with loss incidents observed under different network conditions. To do that, we used packet voice traces collected from the experimental testbed setup in $\langle\underline{16}>$. The terminals ran H.323 software configured to use G.711 codec operating at $64 \mathrm{kbps}$. The packet voice traces were captured in the following mobile network conditions and scenarios reflecting today's communication environments, namely mobility, congestion and dynamics:

- Roaming: Many handover instances between wireless AP (Access Point) caused by the user's mobility are observed during a PVC. In that case, no background traffic is added in order to emphasize on mobility effects.

- UDP: A PVC is delivered via a wireless AP loaded by a small and persistent number of UDP flows. Hence, packet voice flows will be delivered via heavily congested links. The user position is maintained static.

- BitTorrent: A PVC is delivered via a wireless AP loaded by BitTorrent client that creates a high number of TCP flows in order to deliver in parallel all chunks composing a given file. This induces a considerable 


\begin{tabular}{|c|c|c|c|c|c|c|c|c|c|}
\hline Network condition & $n c$ & $\bar{s}$ & $p_{u}$ & $p_{c}$ & $p_{n}$ & $\overline{L L I}$ & $\overline{I L D}$ & $V_{\text {LLI }}$ & $V_{\mathrm{ILD}}$ \\
\hline BitTorrent & 10 & 152.522 & 0.268 & 0.303 & 0.265 & 0.033 & 0.865 & 0.319 & 0.137 \\
\hline UDP & 10 & 156.398 & 0.020 & 0.265 & 0.006 & 0.030 & 2.176 & 0.078 & 3.340 \\
\hline Roaming & 12 & 158.16 & 0.081 & 0.984 & 0.080 & 5.300 & 44.28 & 3.359 & 18.687 \\
\hline \multicolumn{10}{|c|}{$n c$ is the number of calls and $\bar{s}$ is average session duration in seconds } \\
\hline$\overline{L L I}, \overline{I L D}, V_{\mathrm{LLI}}$ & & (0) &. & nds & & & & & \\
\hline
\end{tabular}

Table 6: The characteristics of collected VoIP traces.

charge on the queue, CPU, and memory of forwarding nodes.

Table 6 summarizes statistics about the VoIP traces. The packet interval is set to 30 msec. Fig. 5 shows a typical packet loss variation over time observed for each considered network situation. As we can see, BitTorrent conditions induce a highly unstable packet loss process caused mainly by TCP transport protocol behavior that seeks aggressively to occupy all available channel capacity. UDP conditions induce a lightly congested network where packet loss is small. In the Roaming situation, we see long blackout times during a handover that induce a complete blocking of network service. The loss incidents are less frequent in the roaming situation than under the other conditions.

\subsection{The sample length estimate for a faithful Markov loss model}

The established expressions for computing loss metrics are in theory applicable for received/lost distributions with an infinite length. In practice, traces are finite, but assumed sufficiently large. We think, however, that it is crucial to identify the minimal sample length and time required in order to get a representative Markov loss model. To do that, we apply the statistical inference method that estimates, among others, populationscale proportions using sample-scale ones. In addition, we apply Konrad method $\langle 24>$ that was specifically developed to solve this particular issue. 


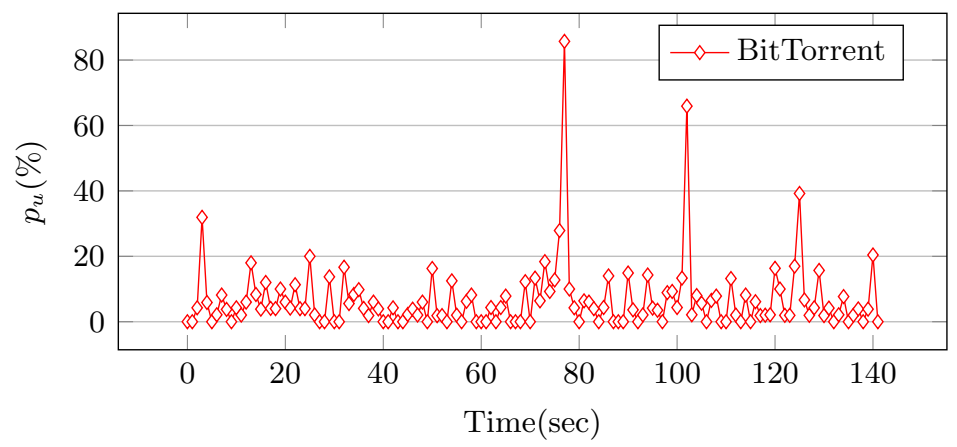

(a) BitTorrent condition

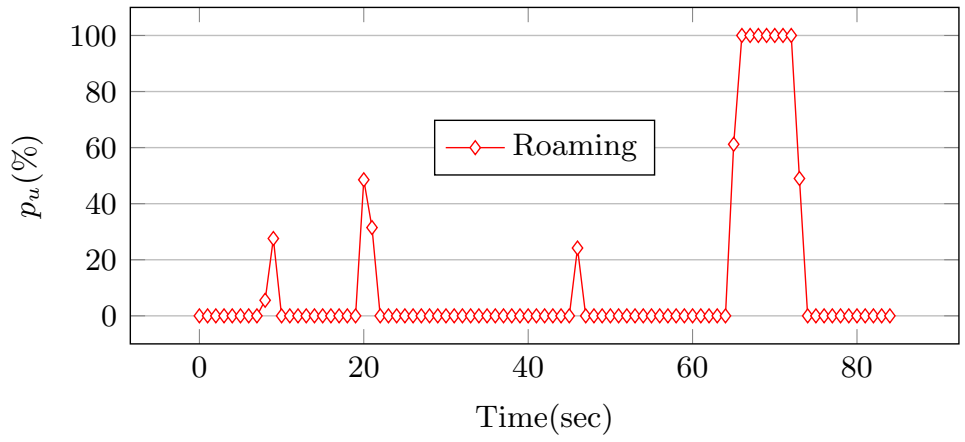

(b) Roaming condition

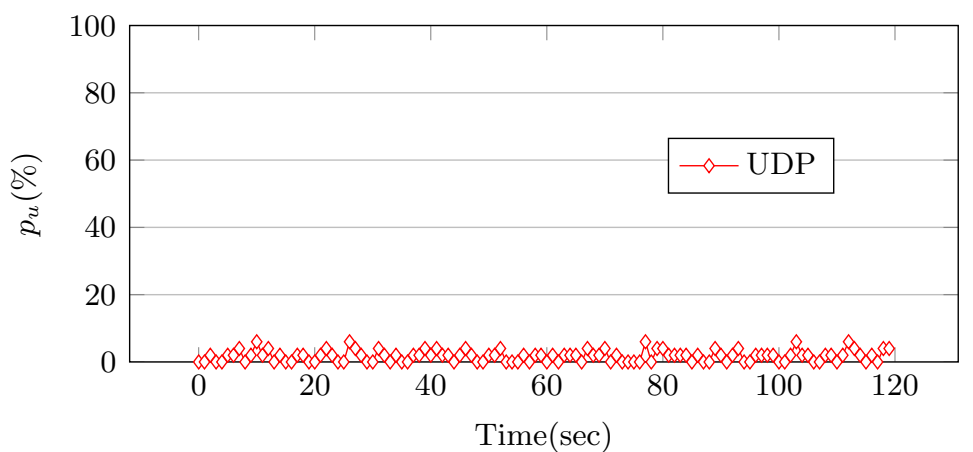

(c) UDP condition

Figure 5: The packet loss process variation under different conditions 


\subsubsection{The statistical inference method}

The ultimate goal is to get a reliable estimation of the transition probability from state $i$ to $j, p_{i, j}$, using a direct edge. This solution has been previously explored in $\langle 5\rangle$. The minimum sample length $s_{i, j}$ required is given by

$$
s_{i, j}=\hat{p}_{i, j}\left(1-\hat{p}_{i, j}\right)\left(\frac{z_{\alpha / 2}}{b}\right)^{2}
$$

where $\hat{p}_{i, j}$ is an estimate of $p_{i, j}$ calculated over an empirical sample, $b$ is the error bound, and $z_{\alpha / 2}$ is the $z$-score obtained from the standard Normal distribution. The confidence interval level is equal to $100(1-\alpha) \%$. This means that the required sample length, $s_{i}$, at state $i$, is equal to the maximum of

all $s_{i, j}$ values. Hence, the total length needed to calibrate our Markov loss model is given by: $\sum_{i=1}^{m} \max _{j}\left\{s_{i, j}\right\}$.

The value of $\hat{p}_{i, j}$ is set to 0.5 when no estimates are available $\langle 25\rangle$. Nevertheless, these values may be retrieved from a pre-built local or remote database, as a function of the identified path and workload characteristics. As we can see, the sample length depends on the number of states $m$. As an example, for an error bound and confidence interval set respectively to \pm 20 and $95 \%$, the resulting calibration delay is equal to $\approx 37 \mathrm{sec}$.

\subsubsection{Konrad method}

Konrad method was specifically developed for estimating the minimum packet loss length required for getting reliable loss metrics from an arbitrary Markov loss model $\langle 24\rangle$. In short, it consists of creating a reference trace of size rlen by concatenating all captured traces. It is divided into subtraces of sizes $r l e n / 2^{j}, j=1,2,3, \ldots, m x$ where the maximum value $m x$ is chosen 


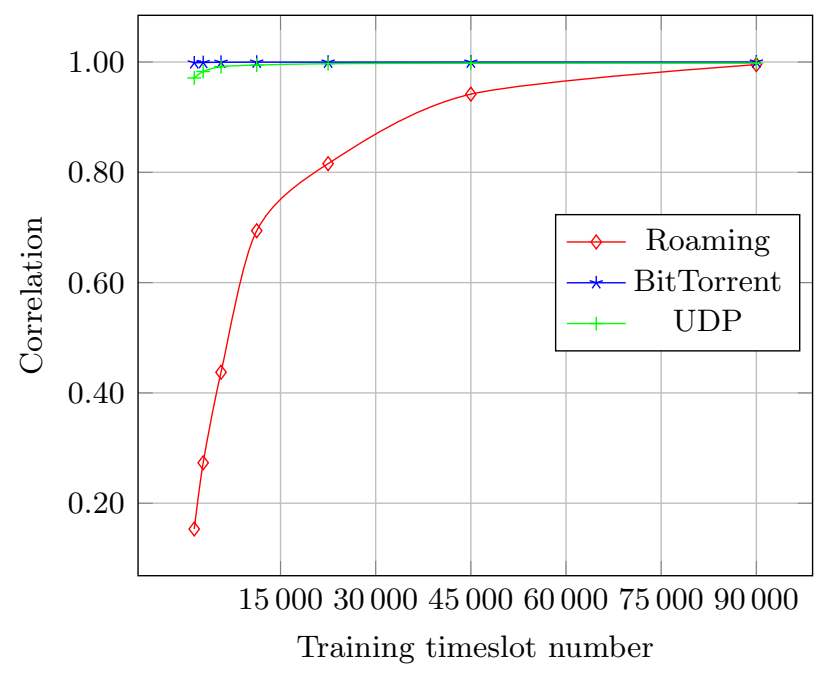

Figure 6: The effect of trace length on model accuracy

in a way that $r l e n / 2^{j}>1.000$ frames.

In our case, we create a reference trace under each condition composed approximately of 180.000 slots that corresponds to half an hour of data. This yields a maximum value $m x$ that is equal to 7 . The ability of a shortened trace to capture all characteristics of a reference trace is realized based on the correlation coefficient, $c c$, relating LLI's CDF of reference and shortened traces $\langle 24\rangle$. A $c c$ of 1 signifies that the compared traces are statistically similar, whereas a $c c$ of 0 implies no statistical similarity. The final $c c$ value of a given trace length is equal to the average of all resulting $c c$ for that packet loss distribution.

The curves given in Fig. 6 show the ability of each trace length to consider all characteristics of a reference trace under different conditions. As we can see, features of packet loss behavior under Bittorrent and UDP conditions may be captured by a packet loss trace composed of 3000 slots. However, we notice that a long packet loss trace composed of 90000 slots is required under 
the Roaming condition. This comes from the distribution of infrequent and long loss incidents resulting in spiky CDFs, which are difficult to follow over a short time interval. This observation has been seen in $\langle 24\rangle$ suggesting using twice the maximum value of ILD that is equal to $18 \mathrm{sec}$ in our case. As such, a safe length should be set to $36 \mathrm{sec}$, in harmony with our preceding analysis.

\subsection{The validation of our Markov loss model}

This section validates the ability of our Markov loss model to capture all statistical characteristics of loss distributions collected under different conditions. The diagram given in Fig. 7 shows the followed validation workflow. As we can see, the set of raw packet loss distributions goes through a pre-processing module that will randomly concatenate all individual distributions. Next, it is transformed from packet to slot level, which depends on the duration $T$. The resulting slot level loss distribution is given to a module that will split the original dataset into training and validation sets, $\mathrm{A}$ and $\mathrm{B}$. Based on our analysis described in the preceding section, the length of training dataset $\mathrm{A}$ is assigned to rlen $/ 2^{7}$ for the Bittorrent and UDP conditions and $r l e n / 2^{1}$ for the Roaming condition, where rlen is approximately equal to 180,000 . The dataset $\mathrm{A}$ is given to a calibration module that identifies different loss periods and compute the transition matrix. The latter is used in order to generate 10 datasets of synthetic losses with a length equal to the loss validation set B. Finally, we analyze the correlation between synthetic and validation datasets.

The histogram plotted in Fig. 8 summarizes $c c$ value under each condition. As we can see, except for LLI under Roaming condition, pretty acceptable $c c$ values, greater than 0.94 , are obtained for all conditions. In 


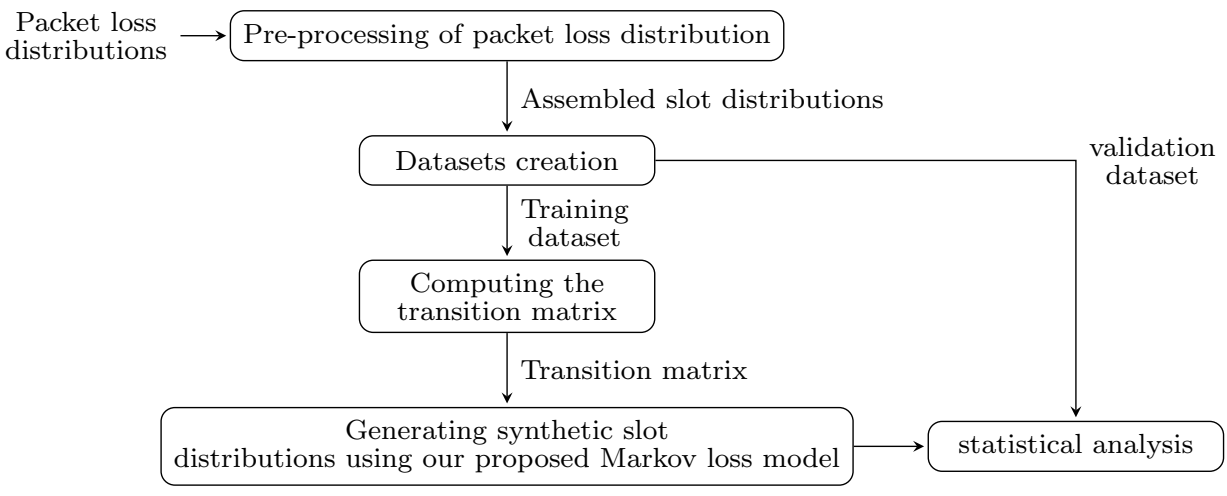

Figure 7: The evaluation framework of our proposed Markov loss model

order to better shows the model performance, we plot in Fig. 9 the CDF of LLI and ILD of validation and synthetic loss distributions under each condition. As we can observe, synthetic traces generally succeed to capture the trends of measured LLI and ILD. The issue is only observed for the CDF of LLI under Roaming where transition steps are abrupt, infrequent and last for a long time. In such a case, the instant when a loss incident occurs within a slot loss distribution becomes crucial. This feature is actually not supported by our model, as loss incidents are randomly inserted. The behavior of synthetic and validation datasets under the Roaming condition are shown in Fig. 10.

As a final step, we give in Table 7 the characteristics of validation and synthetic datasets. As we can see, metrics gathered from the synthetic and validation traces are pretty close. This illustrates the accuracy of our Markov loss model to capture loss metrics at different levels. 


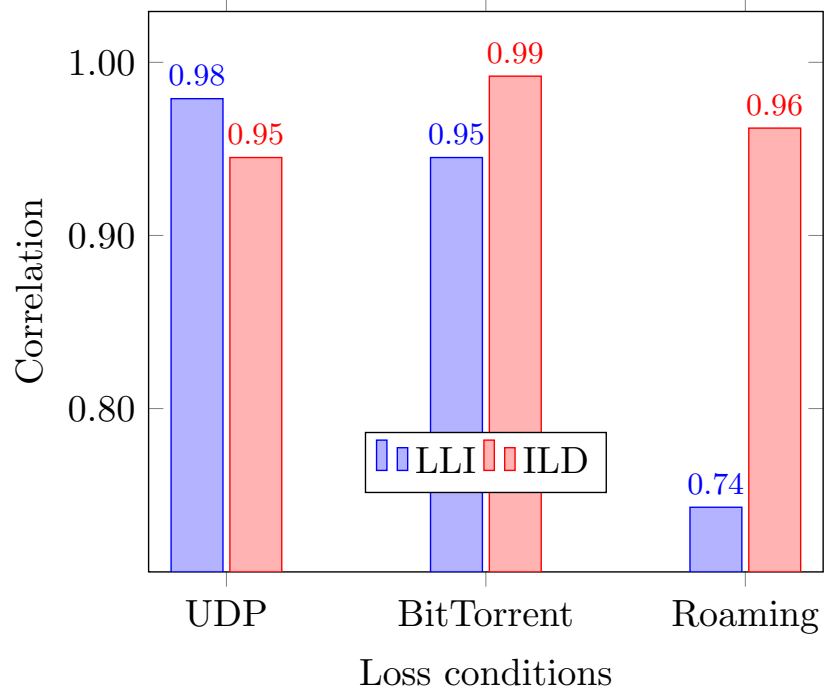

Figure 8: The correlation between synthetic and validation datasets

\begin{tabular}{c||c|c|c|c|c|c|c|c} 
& dataset & $p_{u}$ & $p_{n}$ & $p_{c}$ & $\overline{I L D}$ & $V_{\text {ILD }}$ & $\overline{L L I}$ & $V_{\text {LLI }}$ \\
\hline \multirow{2}{*}{ Roaming } & Validation & 0.095 & 0.095 & 0.99 & 39.37 & 41.80 & 4.34 & 7.64 \\
& Synthetic & 0.067 & 0.0673 & 0.999 & 58.46 & 45.92 & 4.53 & 5.40 \\
\hline \multirow{2}{*}{ BitTorrent } & Validation & 0.262 & 0.247 & 0.94 & 0.12 & 1.94 & 0.04 & 0.69 \\
& Synthetic & 0.244 & 0.234 & 0.945 & 0.14 & 0.46 & 0.05 & 0.19 \\
\hline \multirow{2}{*}{ UDP } & Validation & 0.02 & 0.02 & 1.00 & 2.04 & 2.18 & 0.04 & 0.20 \\
& Synthetic & 0.02 & 0.01 & 1.00 & 2.00 & 1.65 & 0.04 & 0.04 \\
\hline
\end{tabular}

Table 7: The comparison between original and synthetic VoIP traces. 


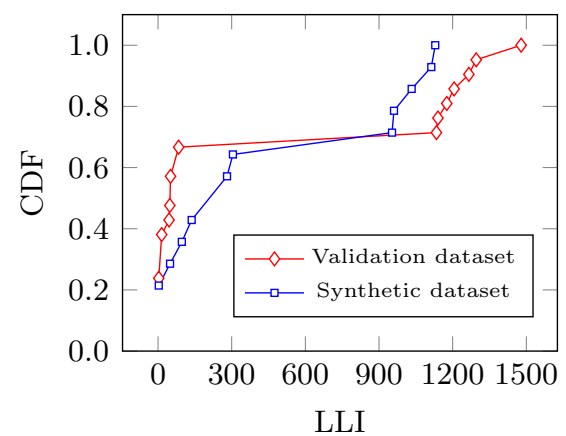

(a) CDF of LLI under roaming

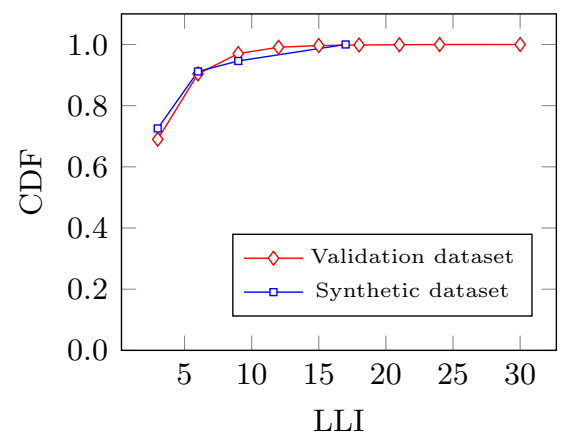

(c) CDF of LLI under BitTorrent

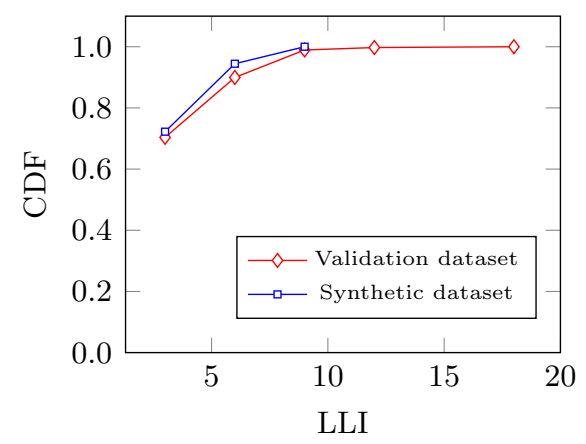

(e) CDF of LLI under UDP

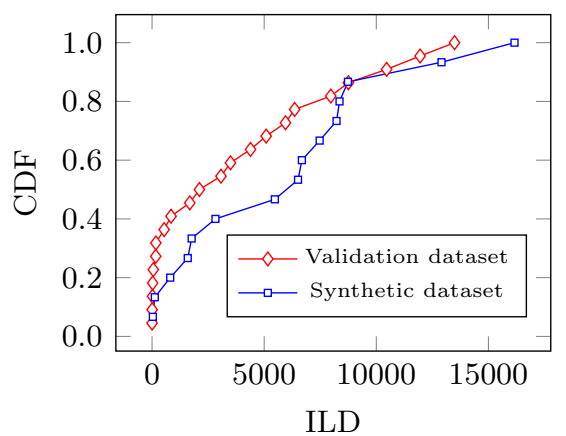

(b) CDF of ILD under roaming

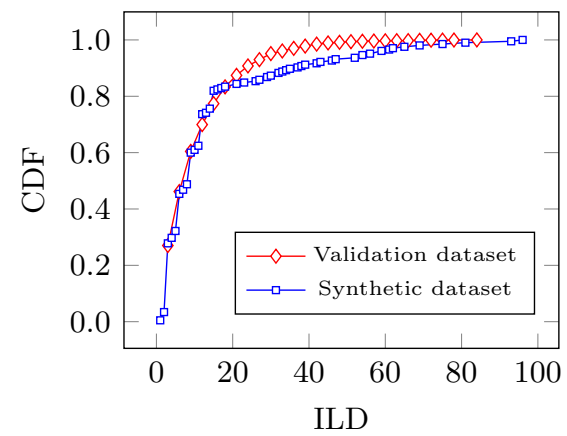

(d) CDF of ILD under BitTorrent

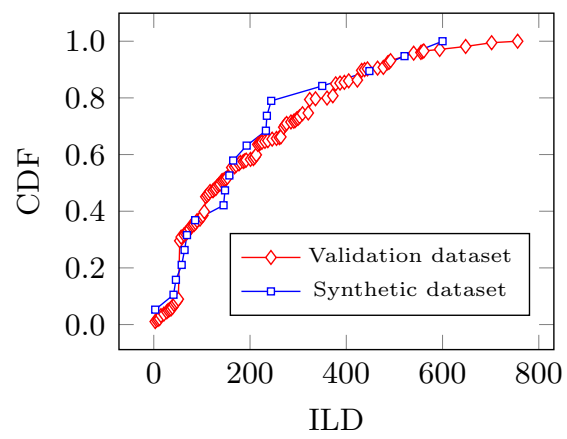

(f) CDF of ILD under UDP

Figure 9: The packet loss process variation under different conditions 


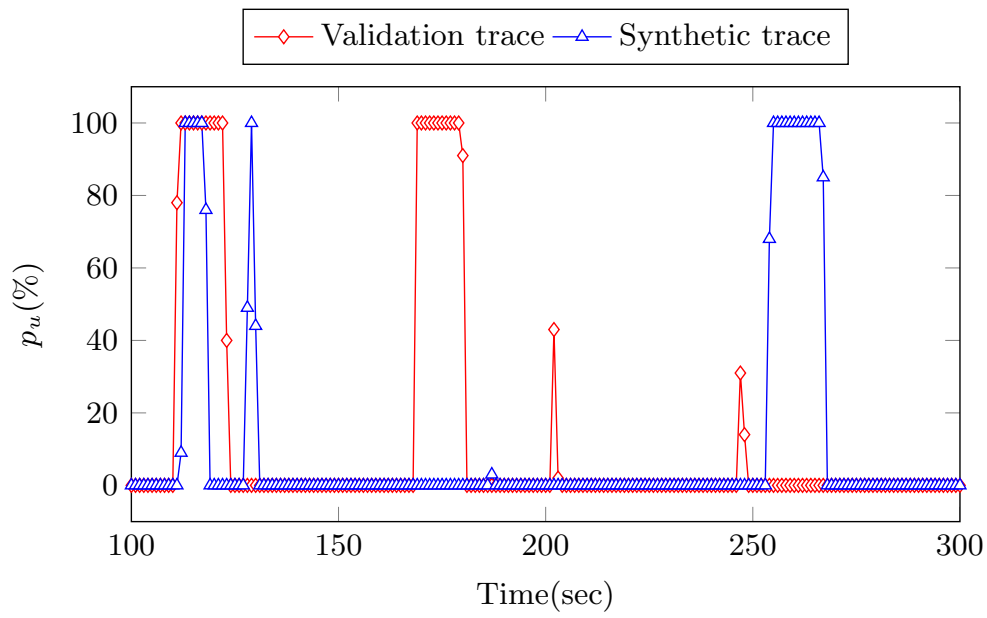

Figure 10: The validation and synthetic roaming behavior

\section{Conclusions and perspectives}

This paper described a perception-oriented Markov loss model that is able to (1) consider packet time intervals, (2) differentiate between isolated, low and high frequency short LLI and interruption periods, and (3) account for long and short-term trends of observed loss incidents. The Markov model considers loss processes at global, channel and period levels. It is used in order to obtain characterization metrics of loss processes using closed-form expressions. The associated numerical analysis shows that our model is able to capture accurately loss behavior under different network conditions.

As future work, we plan to develop quality models that accept as parameters our designed and proposed characterization metrics. Moreover, we plan to extend our model in order to include the features of removed and played voice packets, such as voiced or unvoiced. Furthermore, we believe that considering cognitive and linguistic aspects should be investigated for a better characterization of loss incidents on an user-by-user basis. 


\section{Acknowledgment}

We would like to thank Ilias Tsompanidisin for providing us VoIP traces used for evaluating and validating our loss models.

\section{References}

[1] S. Jelassi, G. Rubino, H. Melvin, H. Youssef, G. Pujolle, Quality of Experience of VoIP Service: A Survey of Assessment Approaches and Open Issues, IEEE Communications Surveys and Tutorials 14 (2) (2012) pp. 491-513.

[2] J.-C. Bolot, H. Crépin, A. V. Garcia, Analysis of Audio Packet Loss in the Internet, in: Proc. of 12th Workshop on Network and Operating Systems Support for Digital Audio and Video (NOSSDAV '95), Durham, New Hampshire, USA, 1995, pp. 154-165.

[3] R. Koodli, C. Krishna, Noticeable loss: A metric for capturing loss pattern for continuous media applications, in: Proc. of Internet routing and quality of service, San Francisco, CA, USA, 1993.

[4] H. Sanneck, G. Carle, A Framework Model for Packet Loss Metrics Based on Loss Runlengths, in: Proc. of of the SPIE/ACM SIGMM Multimedia Computing and Networking Conference 2000 (MMCN 2000), San Jose, CA, USA, 2000, pp. 177-187.

[5] M. Yajnik, S. B. Moon, J. Kurose, D. Towsley, Measurement and Modelling of the Temporal Dependence in Packet Loss, in: Proc. of IEEE INFOCOM 1999, New York, NY, 1999, pp. 345-352.

[6] W. Jiang, H. Schulzrinne, Modeling of Packet Loss and Delay and their 
Effect on Real-Time Multimedia Service Quality, in: Proc. of IEEE NOSSDAV' 2000, Chapel Hill, North Carolina, USA, 2000, p. 55.

[7] A. Clark, Modeling the Effects of Burst Packet Loss and Recency on Subjective Voice Quality, in: Proc. of 2nd IP-Telephony Workshop (IPTel'2001), Columbia University, New York City, USA, 2001, pp. 123127.

[8] C. Boutremans, G. Iannaccone, C. Diot, Impact of link failures on VoIP performance, in: Proc. of IEEE NOSSDAV' 2002, Miami Beach, FL, USA, 2002, pp. 63-71.

[9] N. Kushman, S. Kandula, D. Katabi, Can You Hear Me Now?! It Must Be BGP, ACM SIGCOMM Computer Communication Review 37 (2007) pp. 77-84.

[10] L. Roychoudhuri, E. Al-Shaer, G. B. Brewster, On the impact of loss and delay variation on Internet packet audio transmission, Computer Communications 29 (2006) pp. 1578-1589.

[11] A. Markopoulou, G. Iannaccone, S.Bhattacharyya, C.-N. Chuah, Y. Ganjali, C. Diot, Characterization of failures in an operational IP backbone network, IEEE/ACM Transactions of Networking 16 (2008) pp. 749-762.

[12] J. Wang, S. Lian, W. Dong, X.-Y. Li, Y. Liu, Every Packet Counts: Loss and Reordering Identification and Its Application in Delay Measurement, IEEE/ACM Transactions on Networking 24 (6) (2016) pp. $3426-3438$. 
[13] V. Paxson, End-to-End routing behavior in the Internet, IEEE/ACM Transactions of Networking 5 (1997) pp. 601-615.

[14] A. Lakhina, M. Crovella, C. Diot, Diagnosing network-wide traffic anomalies, in: Proc. of IEEE SIGCOMM 2004, Portland, OR, USA, 2004, pp. 219-230.

[15] N. Majed, S. Ragot, X. Lagrange, A. Blanc, Delay and quality metrics in Voice over LTE (VoLTE) networks: An end-terminal perspective, in: Proc. of International Conference on Computing, Networking and Communications (ICNC 2017), Silicon Valley, USA, 2017, pp. pp. 643648.

[16] I. Tsompanidis, G. Fortetsanakis, T. Hirvonen and M. Papadopouli, Analyzing the impact of various wireless network conditions on the perceived quality of VoIP, in: Proc. of 17th IEEE Workshop on Local and Metropolitan Area Networks (LANMAN'2010), Long Branch, NJ, USA, 2010, pp. 1-6.

[17] R. Warren, Auditory Perception: An Analysis and Synthesis, New York: Cambridge University Press, Cambridge University, 2004, Ch. Perceptual restoration of missing sounds, pp. 3917-3923.

[18] M. Yajnik, J. Kurose and D. Towsley, Packet Loss Correlation in the MBone Multicast Network: Experimental Measurements and Markov Chain Models, Tech. Rep. 95-115, UMASS CMPSCI, Department of Computer Science University of Massachusetts at Amherst, MA 01003 (June 1996).

[19] S. Salsano, F. Ludovici, A. Ordine, D. Giannuzzi, Definition of a general 
and intuitive loss model for packet networks and its implementation in the Netem module in the Linux kernel, Tech. rep., University of Rome "Tor Vergata", Via del Politecnico 1, 00133 Rome (August 2012).

[20] C. T. Calafate, P. Manzoni and M. P. Malumbres, Speeding up the evaluation of multimedia streaming applications in MANETs using HMMs, in: Proc. of 7th IEEE/ACM Symposium on Modeling Analysis and Simulation of Wireless and Mobile Systems (MSWiM'2004), Venice, Italy, 2004, pp. 315-322.

[21] W. Jiang and H. Schulzrinne, Perceived Quality of Packet Audio under Bursty Losses, Tech. rep., Department of Computer Science, Columbia University, CUCS-009-01 (2001).

[22] H. Sanneck, Concealment of Lost Speech Packets Using Adaptive Packetization, in: Proc. of IEEE International Conference on Multimedia Computing and Systems, Austin, USA, 1998, pp. 140-148.

[23] S. Tao and R. Guerin, On-line Estimation of Internet Path Performance: An Application Perspective, in: Proc. of of IEEE INFOCOM 2004, Hong Kong, China, 2004, pp. 1774-1785.

[24] A. Konrad, B. Zhao, and A. D. Joseph, Determining Model Accuracy of Network Traces, Elsevier Journal of Computer and System Sciences. Special Issue: Performance Modeling and Evaluation of Computer Systems. 72 (7) (2006) pp. 1156-1171.

[25] R. L. Smith, Basic Concepts of Statistics and Data Analysis (Oct. 2013). URL http://wWw . unc.edu/ rls/s151-2010/s151.html 


\section{A. Appendix : Loss metrics computing algorithms}

This appendix describes algorithms enabling to compute different loss metrics given in Table I. They are directly sampled from a preprocessed distribution of lost/received slots. The preprocessing enables to identify and classify loss periods into isolated, low and high frequency short LD and interruption periods. Algorithm 5 extracts and records the series of interloss and loss incident of a given packet loss distribution. Algorithm 6, 8 and 7 calculate respectively loss metrics at slot, channel and period levels.

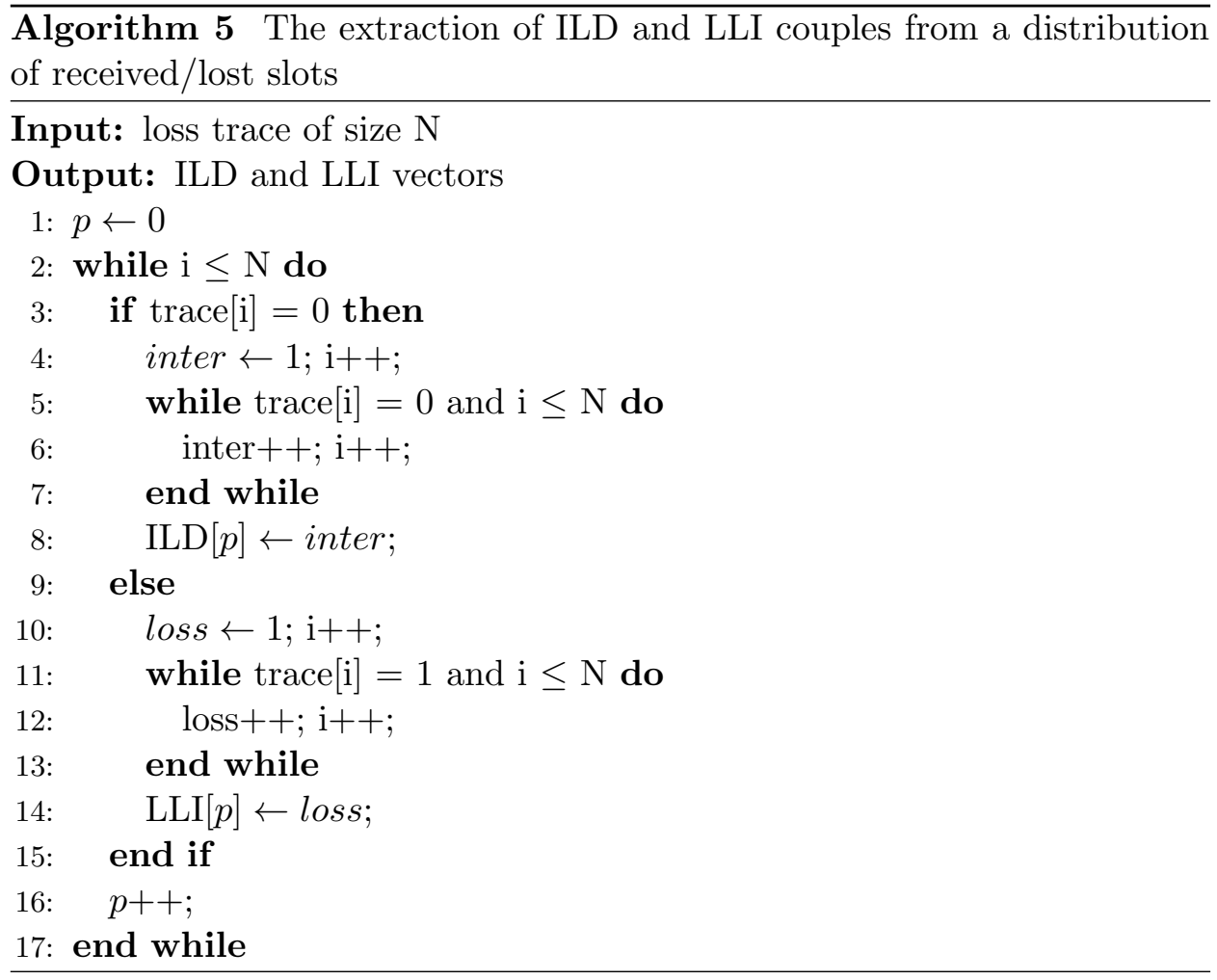




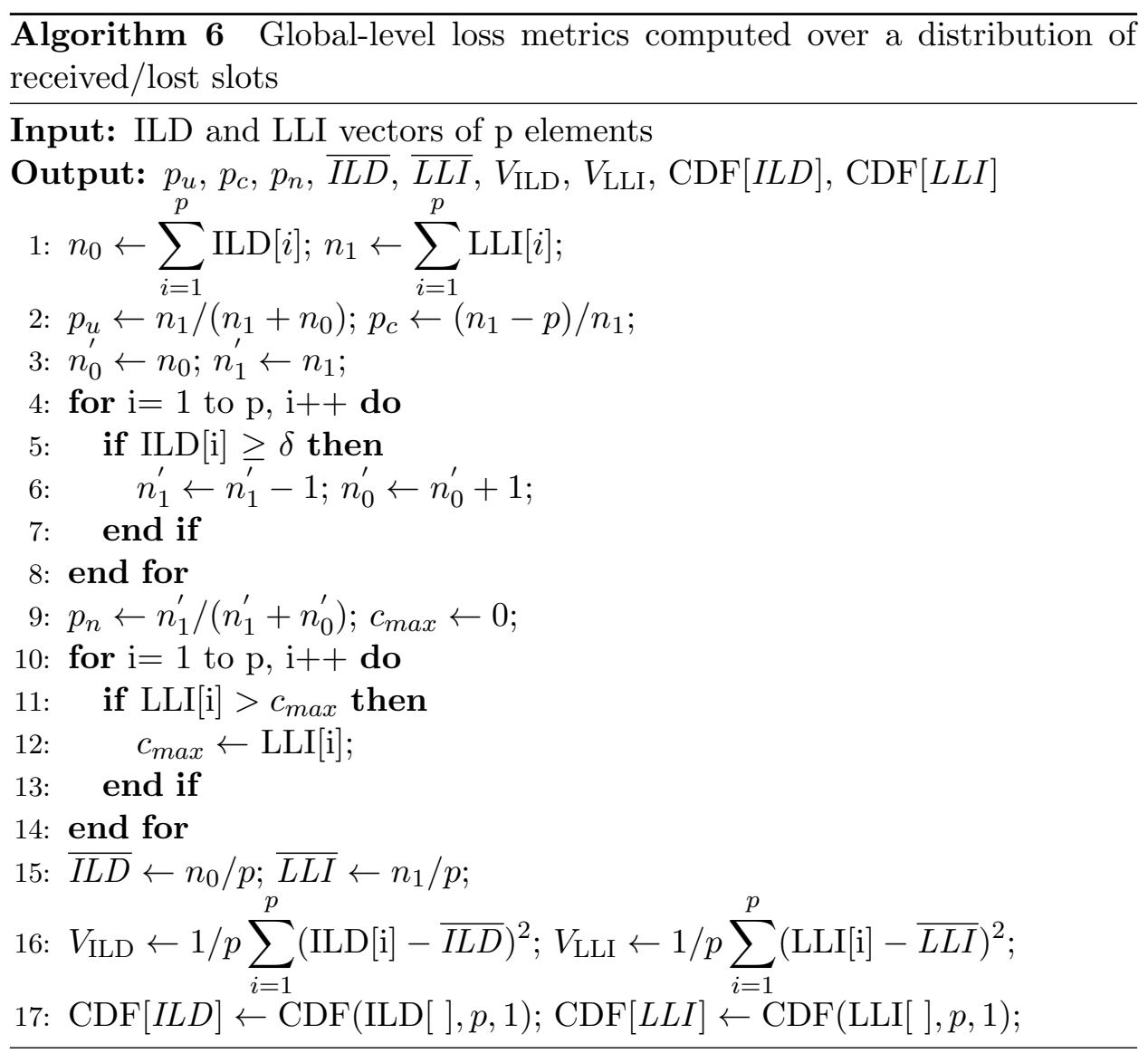




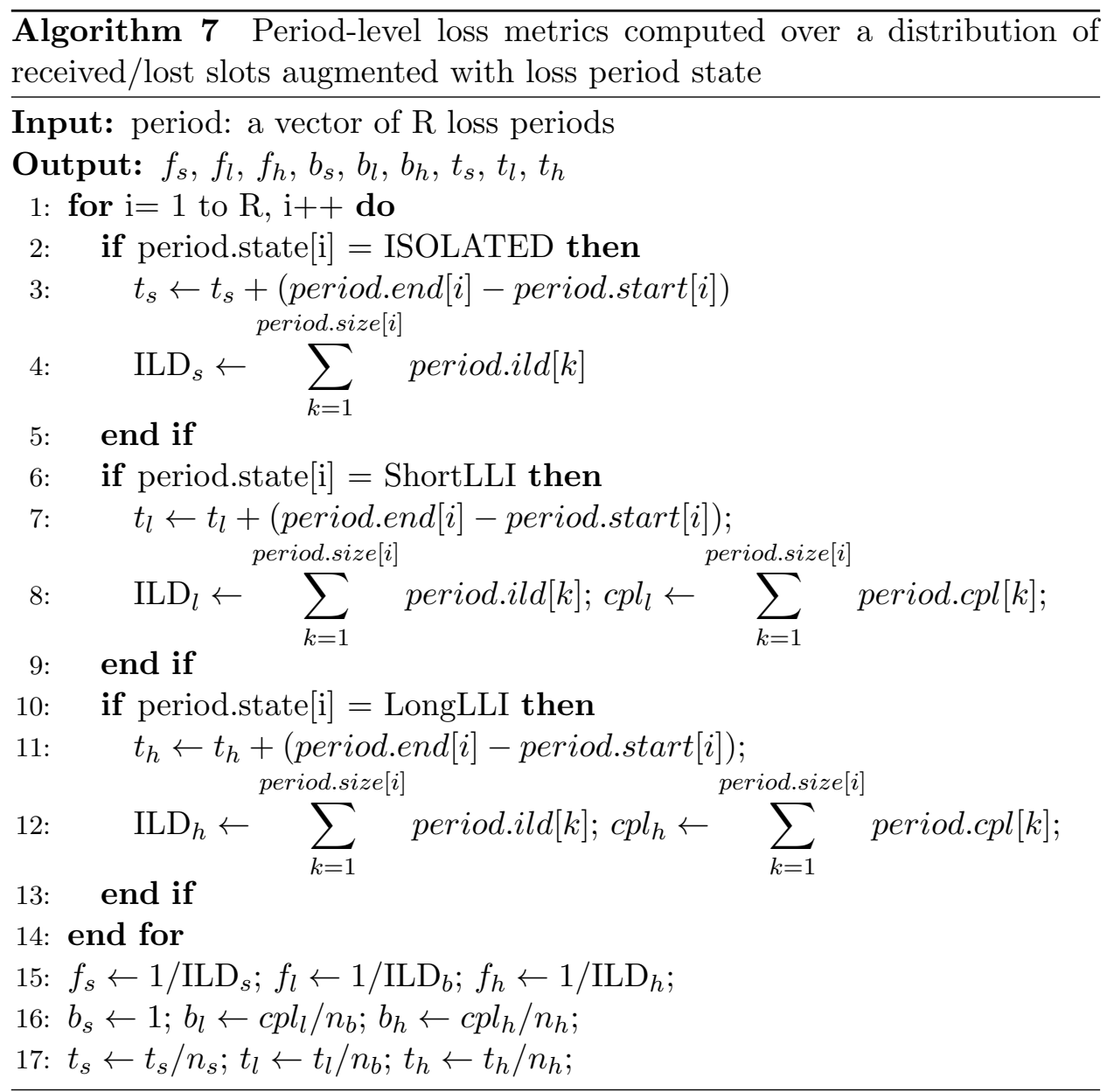




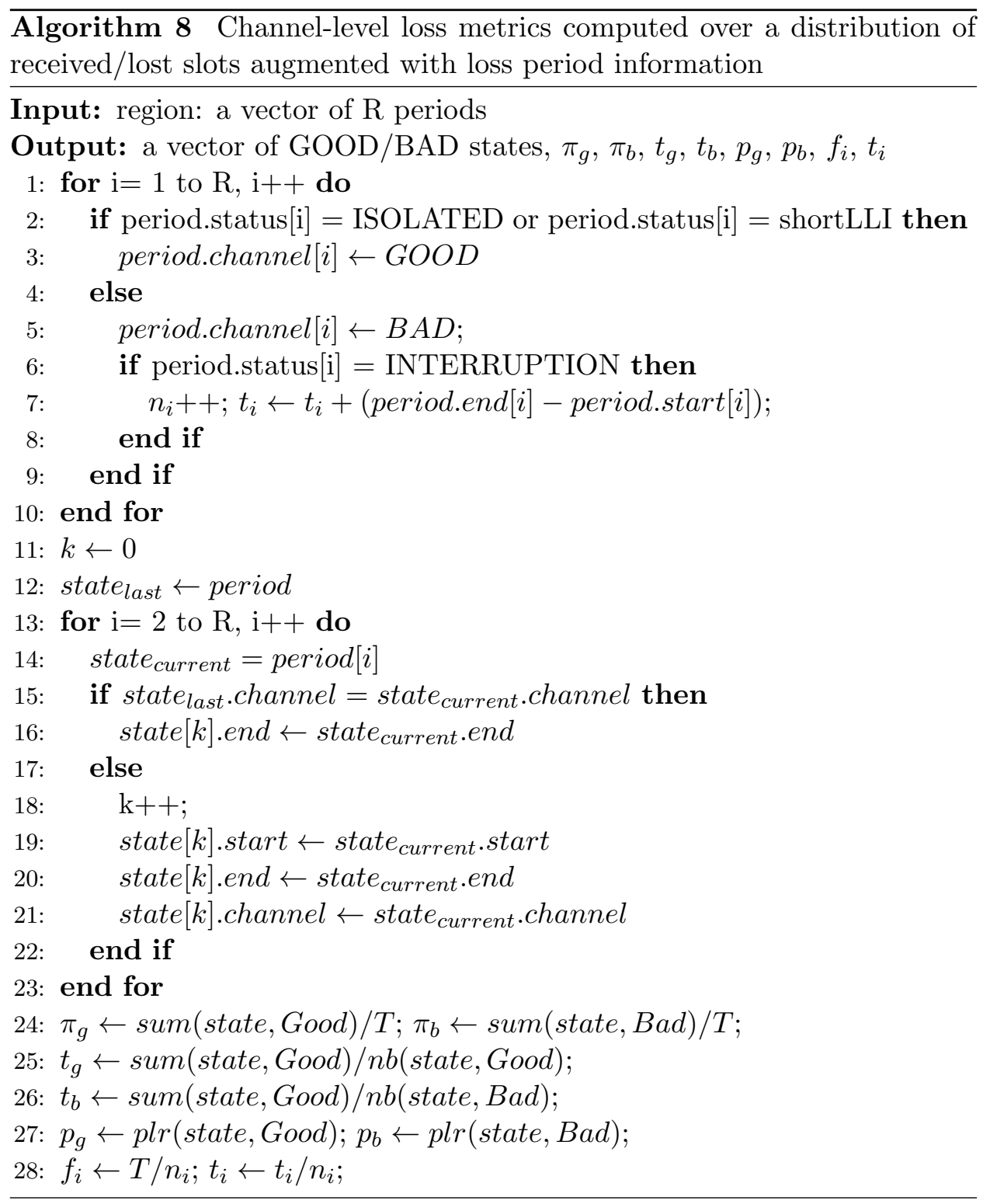

